\title{
Universal algorithms for computing spectra of periodic operators
}

\author{
Jonathan Ben-Artzi ${ }^{1}$ - Marco Marletta ${ }^{1}$ - Frank Rösler ${ }^{1}$ \\ Received: 20 April 2021 / Revised: 11 November 2021 / Accepted: 10 December 2021 / \\ Published online: 14 February 2022 \\ (c) The Author(s) 2022
}

\begin{abstract}
Schrödinger operators with periodic (possibly complex-valued) potentials and discrete periodic operators (possibly with complex-valued entries) are considered, and in both cases the computational spectral problem is investigated: namely, under what conditions can a 'one-size-fits-all' algorithm for computing their spectra be devised? It is shown that for periodic banded matrices this can be done, as well as for Schrödinger operators with periodic potentials that are sufficiently smooth. In both cases implementable algorithms are provided, along with examples. For certain Schrödinger operators whose potentials may diverge at a single point (but are otherwise wellbehaved) it is shown that there does not exist such an algorithm, though it is shown that the computation is possible if one allows for two successive limits.
\end{abstract}

Mathematics Subject Classification 35J10 $\cdot 47 \mathrm{~N} 40 \cdot 68 \mathrm{Q} 25 \cdot 35 \mathrm{P} 05 \cdot 81 \mathrm{Q} 10$

JBA acknowledges support from an Engineering and Physical Sciences Research Council Fellowship EP/N020154/1. MM acknowledges support from an Engineering and Physical Sciences Research Council Grant EP/T000902/1. FR acknowledges support from the European Union's Horizon 2020 Research and Innovation Programme under the Marie Skłodowska-Curie grant agreement No 885904.

The authors thank the anonymous referees for their detailed comments and remarks which helped improve both the quality and presentation of this paper.

$凶$ Jonathan Ben-Artzi

Ben-ArtziJ@cardiff.ac.uk

Marco Marletta

MarlettaM@cardiff.ac.uk

Frank Rösler

RoslerF@cardiff.ac.uk

1 School of Mathematics, Cardiff University, Senghennydd Road, Cardiff, Wales CF24 4AG, UK 


\section{Contents}

1 Introduction and main results f . . . . . . . . . . . . . . . . . . . . . 720

1.1 The solvability complexity index hierarchy . . . . . . . . . . . . . . . . . . . . 722

1.2 Periodic operators . . . . . . . . . . . . . . . . . . . . . . . 724

Organization of the paper . . . . . . . . . . . . . . . . . . . 725

2 The solvability complexity index hierarchy . . . . . . . . . . . . . . . . . 725

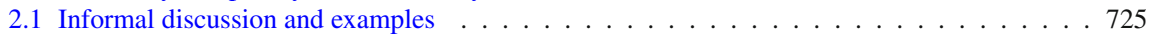

2.2 Definitions . . . . . . . . . . . . . . . . . . 726

3 Banded periodic matrices . . . . . . . . . . . . . . . . . . . . . 728

3.1 Proof of Theorem 1.1(i) . . . . . . . . . . . . . . . . . . . . . . . . 729

3.2 Floquet-Bloch transform . . . . . . . . . . . . . . . . . . . . . 730

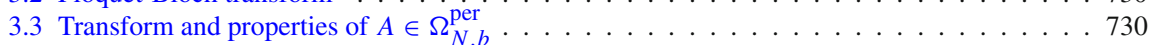

3.4 Proof of Theorem 1.1(ii) . . . . . . . . . . . . . . . . . . . . 734

4 Schrödinger operators with periodic potentials . . . . . . . . . . . . . . . 736

4.1 Estimating and approximating the potential f . . . . . . . . . . . . . . 737

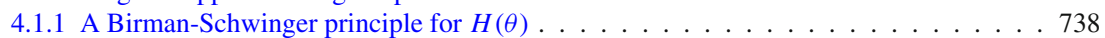

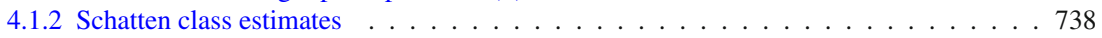

4.1 .3 Approximation of the potential . . . . . . . . . . . . . . . 741

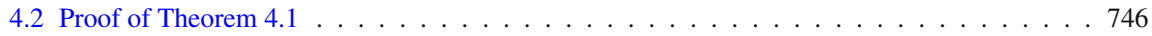

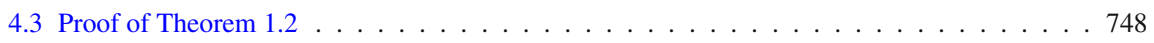

5 Sometimes two limits are necessary . . . . . . . . . . . . . . . . . . . . 750

5.1 Lemmas . . . . . . . . . . . . . . . . . . . . . . . . . . . . . . . . . . . . . . . . . . . . . . . . . . . . .

5.2 Proof of Theorem $5.1 \ldots \ldots \ldots \ldots \ldots \ldots \ldots$

6 Numerical results . . . . . . . . . . . . . . . . . . . . . . . . . . 758

6.1 Implementation in one dimension. The Mathieu equation . . . . . . . . . . . . . . . . 759

6.2 Implementation in two dimensions . . . . . . . . . . . . . . . . . . . . . . . . 761

Appendix A. The Hausdorff and Attouch-Wets distances . . . . . . . . . . . . . . . . . . . . 761

Appendix B. Floquet-Bloch transform . . . . . . . . . . . . . . . . . . . . . . . . 762

Appendix C. Proof of proposition $4.9 \ldots \ldots \ldots \ldots \ldots \ldots$

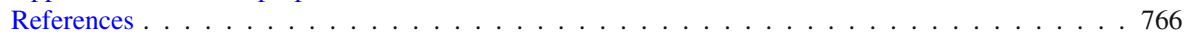

\section{Introduction and main results}

We study the computational spectral problem for periodic discrete operators, acting in $\ell^{2}(\mathbb{Z})$, as well as Schrödinger operators with periodic potentials acting in $L^{2}\left(\mathbb{R}^{d}\right)$. We show that it is possible to compute their respective spectra as limits of finite-dimensional approximations. However, in the Schrödinger case this becomes impossible if the potential is allowed to be discontinuous at a single point (but otherwise it is smooth). More precisely, we prove:

Theorem 1.1 (Periodic banded matrices) Let $B\left(\ell^{2}(\mathbb{Z})\right)$ denote the set of bounded operators on $\ell^{2}(\mathbb{Z})$ and let $\left\{e_{i}\right\}_{i \in \mathbb{Z}}$ be a basis for $\ell^{2}(\mathbb{Z})$. Let $\Omega^{\text {per }} \subset B\left(\ell^{2}(\mathbb{Z})\right)$ be the class of banded, periodic operators with respect to the basis $\left\{e_{i}\right\}_{i \in \mathbb{Z}}$ and $\Omega_{N, b}^{\mathrm{per}}$ the subset of matrices with period $N$ and bandwidth $b$. Then

(i) there exists an algorithm that can compute the spectrum $\sigma(A)$ of any $A \in \Omega^{\mathrm{per}}$ as the limit of a sequence computable approximations;

(ii) there exists an algorithm that can compute the spectrum $\sigma(A)$ of any $A \in \Omega_{N, b}^{\mathrm{per}}$ with guaranteed error bounds. 
Theorem 1.2 (Schrödinger: good case) For $d \in \mathbb{N}$ define the class of potentials

$$
\begin{aligned}
& \Omega^{\mathrm{Sch}}:=\left\{V: \mathbb{R}^{d} \rightarrow \mathbb{C} \mid V\right. \text { is 1-periodic } \\
& \text { and } \left.\left.V\right|_{(0,1)^{d}} \in W^{1, p}\left((0,1)^{d}\right) \text { for some } p>d\right\},
\end{aligned}
$$

and given $M>0$ and $p>d$ define the class

$$
\Omega_{p, M}^{\mathrm{Sch}}:=\left\{V \in \Omega^{\mathrm{Sch}} \mid\|V\|_{W^{1, p}\left((0,1)^{d}\right)} \leq M\right\} .
$$

Then

(i) there exists an algorithm that can compute the spectrum $\sigma(H)$ of any Schrödinger operator $H=-\Delta+V$ with $V \in \Omega^{\mathrm{Sch}}$ as the limit of a sequence computable approximations;

(ii) moreover, for $V \in \Omega_{p, M}^{\mathrm{Sch}}$ this algorithm yields spectral inclusion with guaranteed error bounds;

(iii) if, in addition to belonging to $\Omega_{p, M}^{\mathrm{Sch}}$ the potential $V$ is also real-valued and of bounded variation in the sense of Hardy and Krause, then $\sigma(H)$ can be computed with full error control.

Theorem 1.3 (Schrödinger: bad case) Let $x_{0} \in[0,1]$ and let

$$
\begin{gathered}
\Omega_{x_{0}}^{\mathrm{Sch}}:=\left\{V: \mathbb{R} \rightarrow \mathbb{R} \mid V \text { is 1-periodic, } V\left(x_{0}\right)=0\right. \\
\text { and } \left.\left.V\right|_{[0,1]} \in L^{2}([0,1]) \cap C^{\infty}\left([0,1] \backslash\left\{x_{0}\right\}\right)\right\} .
\end{gathered}
$$

Then there does not exist an algorithm that can compute the spectrum $\sigma(H)$ of any Schrödinger operator $H=-\Delta+V$ with $V \in \Omega_{x_{0}}^{\text {Sch }}$ as the limit of a sequence computable approximations. However, there does exist an algorithm that can compute $\sigma(H)$ by taking two successive limits.

Below, in Sect. 2, we give precise definitions of what an 'algorithm' is, what information is available to it, how it computes, and hence what we mean when we say 'computable'. Informally, Theorems 1.1 and 1.2 imply that these computations can be performed numerically. In fact, we provide actual algorithms which can access the matrix entries (in Theorem 1.1) and pointwise evaluations of the potential (in Theorem 1.2). This should be contrasted with Theorem 1.3 which implies that it is impossible to devise an algorithm that can compute the spectrum of any Schrödinger operator with a potential belonging to $\Omega_{x_{0}}^{\mathrm{Sch}}$. We prove this by contradiction: assuming the existence of such an algorithm, we explicitly construct a potential $V \in \Omega_{x_{0}}^{\text {Sch }}$ for which this algorithm would fail in its attempt to compute the spectrum.

These statements are nontrivial. The existence of a 'one-size-fits-all' algorithm as in Theorems 1.1 and 1.2 is not obvious: while there are techniques for computing the spectrum of a given operator in each of the above cases-these are indeed wellstudied problems - here we prove the existence of a single algorithm (which can be coded) that can handle any input from the given class, without any additional a priori information. Moreover, in the cases of $\Omega_{N, b}^{\mathrm{per}}$ and $\Omega_{p, M}^{\mathrm{Sch}}$ there are even guaranteed error 
bounds. In particular, even when only finitely many values of the potential are known to the algorithm, it can still produce an approximation, which contains the original spectrum up to a known error. Conversely, the non-existence result of Theorem 1.3 is also not obvious: we prove that regardless of what operations are allowed, as long as the algorithm can only read a finite amount of information at each iteration, there will necessarily be a potential for which the computation will fail. In the spirit of the Solvability Complexity Theory (see a brief discussion below in Subsection 1.1, with more details in Sect. 2) we show that if one allows for two successive limits (which cannot be collapsed to a single limit), the computation is possible. Note that although the class of potentials $\Omega_{x_{0}}^{\text {Sch }}$ allows for a blowup near $x_{0}$, all potentials in this class are integrable over compact sets, and so $x_{0}$ is still a regular point for the differential equation [10, footnote, p.67]; elsewhere the potentials are even more well-behaved.

\subsection{The solvability complexity index hierarchy}

Our exploration of the spectral computational problem-for both discrete and Schrödinger operators - continues a line of research initiated by Hansen in [21] and then further expanded in $[4,5]$. This sequence of papers established the so-called Solvability Complexity Index (SCI) Hierarchy, which is a classification of the computational complexity of problems that cannot be computed in finite time, only approximated. This theory is motivated by the following general questions:

What are the limitations of computers, and can they be quantified? Do there always exist algorithms to approximate any problem?

Consider, for instance, the problem of computing the spectrum $\sigma(A)$ of an operator $A \in B\left(\ell^{2}(\mathbb{N})\right)\left(B\left(\ell^{2}(\mathbb{N})\right)\right.$ is the set of bounded linear operators on $\left.\ell^{2}(\mathbb{N})\right)$. Any such $A$ can be represented as an infinite matrix. A computer, however, will never have access to the entire matrix: it will always only 'see' finitely many entries of this matrix, and will only be able to perform finitely many operations, leading to some approximation $\sigma_{n}(A)$. In this level of abstraction, can anything be said about the distance of $\sigma_{n}(A)$ from $\sigma(A)$ ? Can we always devise an approximation procedure that will guarantee the convergence of $\sigma_{n}(A)$ to $\sigma(A)$ for any $A \in B\left(\ell^{2}(\mathbb{N})\right)$ ?

The papers [4,21] prove the following remarkable result: there will always be some $M \in B\left(\ell^{2}(\mathbb{N})\right)$ for which $\sigma_{n}(M)$ will not converge to $\sigma(M)$ in any metric. This does not depend on any specific properties of the approximation procedure, as long as it is a procedure that mimics a computer: it can only read a finite amount of information and it works in a consistent manner. However, it is shown that if one allows several successive limits this problem becomes tractable: there does exist an algorithm $\sigma_{n, m, k}$ such that

$$
\lim _{n \rightarrow \infty} \lim _{m \rightarrow \infty} \lim _{k \rightarrow \infty} \sigma_{n, m, k}(A)=\sigma(A), \quad \forall A \in B\left(\ell^{2}(\mathbb{N})\right)
$$

and these limits cannot be exchanged and cannot be collapsed. The parameters $n, m, k \in \mathbb{N}$ are related to how the matrix representation of $A$ is read by the computer and to the size of some thresholds arising in the computation of singular values of the resulting finite sections. This algorithm is given explicitly. 
In this case, we say that the above problem has an SCI value of 3, since 3 successive limits are required. It turns out that if we were to repeat the above discussion with $B\left(\ell^{2}(\mathbb{N})\right)$ replaced by the set of selfadjoint operators in $B\left(\ell^{2}(\mathbb{N})\right)$ then the index would be 2 , and if it were replaced by the set of compact operators in $B\left(\ell^{2}(\mathbb{N})\right)$ then the index would be 1 . Of course, this raises the following fundamental question: what does it mean for an algorithm to require several limits? Naturally, a numerical analyst might answer this question differently to, say, a theoretical computer scientist. However the mathematical fact remains: some computations cannot be carried out using a singlelimit approximation procedure.

The precise definitions relevant to this theory (for instance, a definition of what an algorithm is) are provided in the detailed discussion in Sect. 2. This classification can even be refined further, to account for algorithms that can perform a computation with known error bounds. The following classes of problems can be defined:

$\Delta_{k}:$ For $k \geq 2, \Delta_{k}$ is the class of problems that require at most $k-1$ successive limits to solve, i.e. the class of problems that have an SCI value of at most $k-1$. Problems in $\Delta_{1}$ can be solved in one limit with known error bounds.

$\Sigma_{k}$ : For all $k \in \mathbb{N}, \Sigma_{k} \subset \Delta_{k+1}$ is the class of problems in $\Delta_{k+1}$ that can be approximated from "below" with known error bounds.

$\Pi_{k}:$ For all $k \in \mathbb{N}, \Pi_{k} \subset \Delta_{k+1}$ is the class of problems in $\Delta_{k+1}$ that can be approximated from "above" with known error bounds.

By an approximation from "above" (resp. "below") we mean that the output of the algorithm is a superset (resp. subset) of the object we are computing (this clearly requires that this object and its approximations belong to a certain topological space). It can also be shown that for $k \in\{1,2,3\}$ we have $\Delta_{k}=\Sigma_{k} \cap \Pi_{k}$.

Previous results: In [4] the spectral computational problems for both $B\left(\ell^{2}(\mathbb{N})\right)$ and for Schrödinger operators were addressed. Some of the results shown there include

$$
\begin{array}{ll}
\text { approximating } \sigma(A) \text { for } A \in B\left(\ell^{2}(\mathbb{N})\right) & \in \Pi_{3} \backslash \Delta_{3} \\
\text { approximating } \sigma(A) \text { for } A \in\left\{B \in B\left(\ell^{2}(\mathbb{N})\right) \mid B \text { is selfadjoint }\right\} & \in \Sigma_{2} \backslash \Delta_{2} \\
\text { approximating } \sigma(A) \text { for } A \in\left\{B \in B\left(\ell^{2}(\mathbb{N})\right) \mid B \text { is banded }\right\} & \in \Pi_{2} \backslash \Delta_{2} \\
\text { approximating } \sigma(-\Delta+V) \text { for } V \text { bounded with known } B V \text { bounds }{ }^{1} \in \Pi_{2} \backslash \Delta_{1}
\end{array}
$$

New results: Theorems $1.1,1.2$ and 1.3 respectively imply that
approximating $\sigma(A)$ for $A \in \Omega^{\text {per }}$
approximating $\sigma(A)$ for $A \in \Omega_{N, b}^{\text {per }}$
$\in \Delta_{2}$
approximating $\sigma(-\Delta+V)$ for $V \in \Omega^{\mathrm{Sch}}$
$\in \Delta_{1}$
approximating $\sigma(-\Delta+V)$ for $V \in \Omega_{p, M}^{\mathrm{Sch}}$
$\in \Delta_{2}$
$\in \Pi_{1}$
approximating $\sigma(-\Delta+V)$ for $V \in \Omega_{p, M}^{\mathrm{Sch}}$
and $V$ real-valued $\in \Delta_{1}$
approximating $\sigma(-\Delta+V)$ for $V \in \Omega_{x_{0}}^{\text {Sch }}$
$\in \Delta_{3} \backslash \Delta_{2}$.

\footnotetext{
1 * These known BV bounds mean that for any $R>0$ one has a priori knowledge of the total variation of $\mathrm{V}$ on the ball $B_{R}$ of radius $\mathrm{R}$ centered at the origin.
} 
Remark 1.4 We point out that showing that a problem belongs to $\Pi_{1}, \Sigma_{1}$ or $\Delta_{1}$ is significant, as it shows that the computation can be done with certain guaranteed error bounds.

We finally note that recent years have seen a flurry of activity in this direction with many results classifying various problems within the SCI Hierarchy. We point out $[11,12]$ where some of the theory of spectral computations has been further developed; [32] where this has been applied to certain classes of unbounded operators; [2] where solutions of PDEs were considered; [6,7] where we considered resonance problems; and [13] where the authors give further examples of how to perform certain spectral computations with error bounds.

\subsection{Periodic operators}

Schrödinger equations with periodic potentials have been the subject of study since the earliest days of quantum mechanics, perhaps most famously for the Bethe-Sommerfeld Conjecture [37], which states that the number of gaps in the essential spectrum is finite in dimensions $\geq 2$. After more than seventy years this conjecture was finally proved in complete generality for Schrödinger operators in $\mathbb{R}^{d}$ by Parnovski [27]. In dimensions 2 and 3 the conjecture had already been proved by Popov and Skriganov [28] and Skriganov [36] respectively, and in dimension 4 by Helffer and Mohamed [22].

Beyond these results in mathematical analysis, since the 1990s interest in periodic problems has grown rapidly in the applied analysis and computational mathematics literature, partly driven by models of photonic crystals. These models are typically based on time-harmonic Maxwell equations or upon second order elliptic equations with periodic coefficients. Figotin and Kuchment [16-18] give particularly thorough analyses of some of these models, showing that already in these cases with piecewise constant coefficients the associated operators may possess an arbitrarily large number of spectral gaps.

For a periodic problem with some particular coefficients, often the first question of interest is whether it has any spectral gaps at all. Numerical methods may be used to obtain some preliminary evidence, and are almost always based on the Floquet-Bloch decomposition. The fact that the coefficients are often only piecewise continuous requires substantial effort to be given to adaptive meshing, see Giani and Graham [19], although the continuous variation of the quasi-momentum over the Brillouin zone means that some of the effort can be recycled from one quasi-momentum to the next. Despite the substantial computational cost, Floquet-Bloch techniques can sometimes be used to go beyond preliminary evidence, and have been combined with interval arithmetic to obtain algorithms which yield computer-assisted proofs of existence of spectral gaps for a wide class of problems with coefficients expressed in terms of elementary functions, see Hoang, Plum and Wieners [23]. The literature for periodic problems which are not self-adjoint is much less extensive. For the ODE case, the work of Rofe-Beketov [31] is generally the starting point for any research on this topic.

The discrete case encompasses numerous different directions of research. Typical examples are Toeplitz and Laurent operators [20] or Jacobi operators [38]. Another direction that has gained a great deal of attention in the last two decades is that of 
periodic discrete Schrödinger operators and generalizations thereof. A particular type known as almost Mathieu operator has been shown to exhibit rich spectral behavior (e.g. the spectrum can be a set of non-integer Hausdorff dimension, cf. [1,24]). Beyond this, the literature pertaining to operators that are either not tri-diagonal or not selfadjoint is limited. The best starting point would be the book of Trefethen and Embree [39].

\section{Organization of the paper}

In Sect. 2 we give a brief introduction to the main ideas of the SCI theory. Sections 3, 4 and 5 are dedicated to the proofs of Theorems 1.1, 1.2 and 1.3, respectively. Finally, in Sect. 6 we provide some numerical examples.

\section{The solvability complexity index hierarchy}

The Solvability Complexity Index (SCI) and the SCI Hierarchy provide a unified approach for understanding just how "difficult" it is to approximate infinitedimensional problems (such as computing spectra) starting from finite-dimensional approximations. We start by setting the scene with a concrete example before providing precise abstract definitions.

\subsection{Informal discussion and examples}

Consider the set $\Omega=B\left(\ell^{2}(\mathbb{N})\right)$ of all bounded operators on $\ell^{2}(\mathbb{N})$. Let $\left\{e_{i}\right\}_{i \in \mathbb{N}}$ be the canonical basis. Then any element $A \in \Omega$ is represented by an infinite matrix. Denote by $\Lambda$ the set of all entries in this matrix. Then one could ask:

For any $A \in \Omega$, is it possible to compute its spectrum $\sigma(A)$ as the limit of a sequence of computations $\Gamma_{n}$, where each $\Gamma_{n}$ has access to only finitely many elements of $\Lambda$ and can only perform finitely many arithmetic computations?

Needless to say, the whole point here is that the algorithms $\Gamma_{n}$ are not tailored for this specific element $A$ : they are meant to be able to handle any element $A \in \Omega$. The convergence of $\Gamma_{n}(A)$ to $\sigma(A)$ is made precise by realizing them as elements of the metric space $\mathcal{M}=(\operatorname{cl}(\mathbb{C}), d)$ which comprises all closed subsets of $\mathbb{C}$ endowed with an appropriate metric $d$ (such as the Hausdorff metric).

In [21], Hansen showed that it is possible to compute $\sigma(A)$ for any $A \in \Omega$ as above. However, rather than having algorithms $\Gamma_{n}$ with a single index $n \in \mathbb{N}$, three indices were required, satisfying $\sigma(A)=\lim _{n_{3} \rightarrow \infty} \lim _{n_{2} \rightarrow \infty} \lim _{n_{1} \rightarrow \infty} \Gamma_{n_{1}, n_{2}, n_{3}}(A)$. The algorithms $\Gamma_{n_{1}, n_{2}, n_{3}}$ are given explicitly, and can be implemented numerically (though this raises a philosophical question about what it means to take successive limits numerically). In [4] it was proved that this is optimal: this computation cannot be performed with fewer than 3 limits, and hence we say that this problem has an SCI value of 3 .

The SCI value strongly depends on $\Omega$ : intuitively, if $\Omega$ contains fewer elements, then devising a 'one-size-fits-all' algorithm should be easier. Indeed, if one considers 
$\Omega^{\mathrm{sa}}:=\{A \in \Omega \mid A$ is selfadjoint $\}$ then the SCI value reduces to 2 and for $\Omega^{\mathrm{cpt}}:=$ $\{A \in \Omega \mid A$ is compact $\}$ it further reduces to 1 .

The classification into SCI values can be further refined into a classification that takes into account error bounds. This is the so-called SCI Hierarchy which we describe below.

\subsection{Definitions}

We formalize the foregoing example with precise definitions:

Definition 2.1 (Computational problem) A computational problem is a quadruple $(\Omega, \Lambda, \Xi, \mathcal{M})$, where

(i) $\Omega$ is a set, called the primary set,

(ii) $\Lambda$ is a set of complex-valued functions on $\Omega$, called the evaluation set,

(iii) $\mathcal{M}$ is a metric space,

(iv) $\Xi: \Omega \rightarrow \mathcal{M}$ is a map, called the problem function.

Remark 2.2 In this paper it is often clear what $\mathcal{M}, \Lambda$ and $\Xi$ are, and the important element is the primary set $\Omega$. In this case we may abuse notation and refer to $\Omega$ alone as the computational problem.

Definition 2.3 (Arithmetic algorithm) Let $(\Omega, \Lambda, \Xi, \mathcal{M})$ be a computational problem. An arithmetic algorithm is a map $\Gamma: \Omega \rightarrow \mathcal{M}$ such that for each $T \in \Omega$ there exists a finite subset $\Lambda_{\Gamma}(T) \subset \Lambda$ such that

(i) the action of $\Gamma$ on $T$ depends only on $\{f(T)\}_{f \in \Lambda_{\Gamma}(T)}$,

(ii) for every $S \in \Omega$ with $f(T)=f(S)$ for all $f \in \Lambda_{\Gamma}(T)$ one has $\Lambda_{\Gamma}(S)=\Lambda_{\Gamma}(T)$,

(iii) the action of $\Gamma$ on $T$ consists of performing only finitely many arithmetic operations on $\{f(T)\}_{f \in \Lambda_{\Gamma}(T)}$.

Definition 2.4 (Tower of arithmetic algorithms) Let $(\Omega, \Lambda, \Xi, \mathcal{M})$ be a computational problem. A tower of algorithms of height $k$ for $\Xi$ is a family $\Gamma_{n_{1}, n_{2}, \ldots, n_{k}}: \Omega \rightarrow \mathcal{M}$ of arithmetic algorithms such that for all $T \in \Omega$

$$
\Xi(T)=\lim _{n_{k} \rightarrow \infty} \cdots \lim _{n_{1} \rightarrow \infty} \Gamma_{n_{1}, n_{2}, \ldots, n_{k}}(T)
$$

Definition 2.5 (SCI) A computational problem $(\Omega, \Lambda, \Xi, \mathcal{M})$ is said to have a Solvability Complexity Index (SCI) of $k$ if $k$ is the smallest integer for which there exists a tower of algorithms of height $k$ for $\Xi$. If a computational problem has solvability complexity index $k$, we write

$$
\operatorname{SCI}(\Omega, \Lambda, \Xi, \mathcal{M})=k
$$

If there exists a family $\left\{\Gamma_{n}\right\}_{n \in \mathbb{N}}$ of arithmetic algorithms and $N_{1} \in \mathbb{N}$ such that $\Xi=$ $\Gamma_{N_{1}}$ then we define $\operatorname{SCI}(\Omega, \Lambda, \Xi, \mathcal{M})=0$.

Definition 2.6 (The SCI Hierarchy) The SCI Hierarchy is a hierarchy $\left\{\Delta_{k}\right\}_{k \in \mathbb{N}_{0}}$ of classes of computational problems $(\Omega, \Lambda, \Xi, \mathcal{M})$, where each $\Delta_{k}$ is defined as the collection of all computational problems satisfying: 


$$
\begin{array}{lll}
(\Omega, \Lambda, \Xi, \mathcal{M}) \in \Delta_{0} & \Longleftrightarrow & \operatorname{SCI}(\Omega, \Lambda, \Xi, \mathcal{M})=0, \\
(\Omega, \Lambda, \Xi, \mathcal{M}) \in \Delta_{k+1} & \Longleftrightarrow & \operatorname{SCI}(\Omega, \Lambda, \Xi, \mathcal{M}) \leq k, \quad k \in \mathbb{N},
\end{array}
$$

with the special class $\Delta_{1}$ defined as the class of all computational problems in $\Delta_{2}$ with a convergence rate:

$(\Omega, \Lambda, \Xi, \mathcal{M}) \in \Delta_{1} \Longleftrightarrow \exists\left\{\Gamma_{n}\right\}_{n \in \mathbb{N}}, \exists \epsilon_{n} \downarrow 0$ s.t. $\quad \forall T \in \Omega, d\left(\Gamma_{n}(T), \Xi(T)\right) \leq \epsilon_{n}$.

Hence we have that $\Delta_{0} \subset \Delta_{1} \subset \Delta_{2} \subset \cdots$

When the metric space $\mathcal{M}$ has certain ordering properties, one can define further classes that take into account convergence from below/above and associated error bounds. In order to not burden the reader with unnecessary definitions, we provide the definition that is relevant to the cases where $\mathcal{M}$ is the space of closed (and bounded) subsets of $\mathbb{R}^{d}$ together with the Attouch-Wets (Hausdorff) distance (definitions of which can be found in Appendix A). These are the cases of relevance to us. A more comprehensive and abstract definition can be found in [4].

Definition 2.7 (The SCI Hierarchy (Attouch-Wets/Hausdorff metric)) Consider the setup in Definition 2.6 assuming further that $\mathcal{M}=\left(\operatorname{cl}\left(\mathbb{R}^{d}\right), d\right)$ where $d=d_{\mathrm{AW}}$ or $d=d_{\mathrm{H}}$. Then for $k \in \mathbb{N}$ we can define the following subsets of $\Delta_{k+1}$ :

$$
\begin{aligned}
& \Sigma_{k}=\left\{(\Omega, \Lambda, \Xi, \mathcal{M}) \in \Delta_{k+1} \mid \exists\left\{\Gamma_{n_{1}, \ldots, n_{k}}\right\} \text { s.t. } \forall T \in \Omega, \exists\left\{X_{n_{k}}(T)\right\} \subset \mathcal{M}\right. \text {, s.t. } \\
& \lim _{n_{k} \rightarrow \infty} \cdots \lim _{n_{1} \rightarrow \infty} \Gamma_{n_{1}, \ldots, n_{k}}(T)=\Xi(T) \& \Gamma_{n_{1}, \ldots, n_{k}}(T) \subset X_{n_{k}}(T) \\
&\left.\& d\left(X_{n_{k}}(T), \Xi(T)\right) \leq \epsilon_{n_{k}}\right\}, \\
& \Pi_{k}=\left\{(\Omega, \Lambda, \Xi, \mathcal{M}) \in \Delta_{k+1} \mid \exists\left\{\Gamma_{n_{1}, \ldots, n_{k}}\right\} \text { s.t. } \forall T \in \Omega, \exists\left\{X_{n_{k}}(T)\right\} \subset \mathcal{M},\right. \text { s.t. } \\
& \quad \lim _{n_{k} \rightarrow \infty} \cdots \lim _{n_{1} \rightarrow \infty} \Gamma_{n_{1}, \ldots, n_{k}}(T)=\Xi(T) \& \Xi(T) \subset X_{n_{k}}(T) \\
&\left.\& d\left(X_{n_{k}}(T), \lim _{n_{k-1} \rightarrow \infty} \cdots \lim _{n_{1} \rightarrow \infty} \Gamma_{n_{1}, \ldots, n_{k}}(T)\right) \leq \epsilon_{n_{k}}\right\} .
\end{aligned}
$$

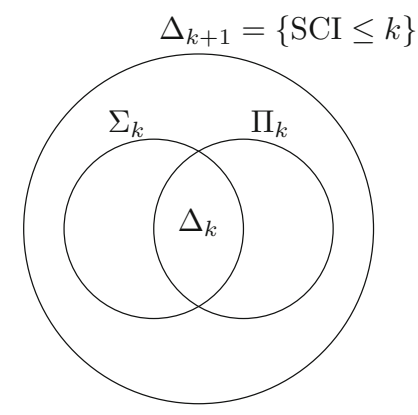

Fig. 1 The SCI Hierarchy for $k \in\{1,2,3\}$ 
It can be shown that $\Delta_{k}=\Sigma_{k} \cap \Pi_{k}$ for $k \in\{1,2,3\}$, see Fig. 1 . We refer to [4] for a detailed treatise.

\section{Banded periodic matrices}

We begin by studying periodic banded matrices, which can be regarded as a natural simplification of periodic Schrödinger operators (indeed, our treatment covers the case of discrete Schrödinger operators). In fact, our method of proof in either case is very similar, but turns out to be considerably simpler in the discrete case and therefore provides a good starting point for our analysis.

In this section we prove Theorem 1.1. We do this by defining an explicit algorithm and show that its output converges to the desired spectrum. The two computational problems we consider only differ in the primary set $\Omega$, and are as follows:

$$
\left\{\begin{aligned}
\Omega & =\Omega^{\text {per }} \text { or } \Omega_{N, b}^{\text {per }} \\
\mathcal{M} & =\left(\{K \subset \mathbb{C} \mid K \text { compact }\}, d_{\mathrm{H}}\right) \\
\Lambda & =\left\{\Omega \ni A \mapsto\left\langle e_{i}, A e_{j}\right\rangle \mid i, j \in \mathbb{Z}\right\} \\
\Xi: & \Omega \rightarrow \mathcal{M} ; \quad A \mapsto \sigma(A),
\end{aligned}\right.
$$

where $\left\{e_{i}\right\}_{i \in \mathbb{Z}}$ denotes the canonical basis of $\ell^{2}(\mathbb{Z})$ and $d_{\mathrm{H}}$ denotes the Hausdorff distance. We remind the reader that $\Omega_{N, b}^{\text {per }}$ is the class of operators on $\ell^{2}(\mathbb{Z})$ whose canonical matrix representation has bandwidth $b$ (i.e. $A_{i j}=0 \forall|i-j|>b$ ) and whose matrix entries repeat periodically along the diagonals with period $N$ (here $N, b \in \mathbb{N}$ ). Clearly, every $A \in \Omega_{N, b}^{\text {per }}$ defines a bounded operator on $\ell^{2}(\mathbb{Z})$. Note that $\Omega^{\text {per }}=$ $\bigcup_{N, b \in \mathbb{N}} \Omega_{N, b}^{\text {per }}$ is the class of operators on $\ell^{2}(\mathbb{Z})$ whose canonical matrix representation is banded and whose matrix entries repeat periodically along the diagonals. In the language of the Solvability Complexity Index, the three parts of Theorem 1.1 can be expressed as follows:

- Part (i) amounts to proving that the computational problem for $\Omega^{\text {per }}$ has an SCI value of 1 (or, equivalently, it belongs to $\Delta_{2}$ ).

- Part (ii) amounts to showing that the computational problem for $\Omega_{N, b}^{\text {per }}$ belongs to $\Delta_{1}$, i.e. it can be approximated with explicit error bounds; this is restated as Theorem 3.3 below.

Remark 3.1 We note that the Hausdorff distance is only defined for non-empty sets, and it is finite only if the sets are bounded. Hence it is important to observe that for any $A \in \Omega^{\text {per }}$, the set $\sigma(A)$ is both non-empty and bounded. Indeed, boundedness of the spectrum follows immediately from boundedness of $A$, while non-emptyness follows from the Floquet-Bloch theory described in Sect. 3.2. We discuss the metrics used in this paper in Appendix A. 
Example 3.2 The class $\Omega_{N, 1}^{\mathrm{per}}$ contains all Jacobi-type matrices of the form

$$
A=\left(\begin{array}{ccccccccc}
\ddots & \ddots & \ddots & & & & & \\
& c_{0} & a_{0} & b_{0} & & & 0 & \\
& & c_{1} & a_{1} & b_{1} & & & \\
& & & \ddots & \ddots & \ddots & & \\
& & & & c_{N-1} & a_{N-1} & b_{N-1} & \\
& 0 & & & c_{0} & a_{0} & b_{0} & \\
& & & & & \ddots & \ddots & \ddots
\end{array}\right) \in \Omega_{N, 1}^{\text {per }} .
$$

\subsection{Proof of Theorem 1.1(i)}

To prove Theorem 1.1(i) we assume to be known Theorem 1.1(ii). Theorem 1.1(ii) can be restated in the language of the SCI Hierarchy as follows:

Theorem 3.3 For any fixed $N, b \in \mathbb{N}$ the computational problem for $\Omega_{N, b}^{\mathrm{per}}$ can be solved in one limit with explicit error bounds, i.e. $\left(\Omega_{N, b}^{\mathrm{per}}, \Lambda, \Xi, \mathcal{M}\right) \in \Delta_{1}$.

The proof of this theorem is contained in Subsection 3.4 below, after some preparatory work. First, however, we prove Theorem 1.1(i):

Proof (Proof of Theorem 1.1(i)) By Theorem 3.3, for every $N \in \mathbb{N}$ there exists a family of algorithms $\left\{\Gamma_{n}^{(N)}\right\}_{n \in \mathbb{N}}$, such that $\Gamma_{n}^{(N)}(B) \rightarrow \sigma(B)$ as $n \rightarrow+\infty$ for any $B \in \Omega_{N, b}^{\text {per }}$. Now, let $A=\left(a_{i j}\right)_{i, j \in \mathbb{Z}} \in \Omega^{\text {per }}$ and define a new family $\left\{\Gamma_{n}\right\}_{n \in \mathbb{N}}$ by the following pseudocode.

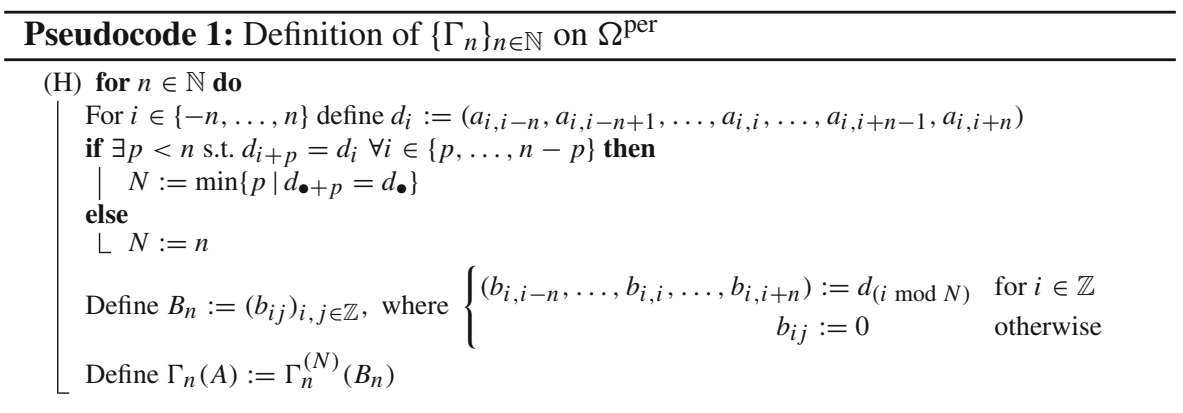

To clarify the meaning of $d_{i}$ we note that in Example 3.2 one would have $d_{i}=$ $\left(\ldots, 0, c_{i}, a_{i}, b_{i}, 0, \ldots\right)$. Loosely speaking, Pseudocode 1 first takes a finite section of $A$, searches it for periodic repetitions, and then defines a matrix $B_{n} \in \Omega_{N, b}^{\text {per }}$ by periodic extension, to which $\Gamma_{n}^{(N)}$ can be applied. Because $A$ is banded and periodic, this routine will eventually find its period: there exists $n_{0} \in \mathbb{N}$ such that for all $n>n_{0}$, 
$N$ (as defined in the routine) is equal to the period of $A$ and $B_{n} \equiv A$. Hence, for $n>n_{0}$ we have $\Gamma_{n}(A)=\Gamma_{n}^{(N)}\left(B_{n}\right)=\Gamma_{n}^{(N)}(A) \rightarrow \sigma(A)$ as $n \rightarrow+\infty$, by the properties of $\Gamma_{n}^{(N)}$.

Finally, note that every line of Pseudocode 1 can be executed with finitely many algebraic operations on the matrix elements of $A$.

The following subsections are devoted to the proof of Theorem 3.3. The proof is constructive, i.e. we will provide an explicit algorithm that computes the spectrum of any given operator $A \in \Omega_{N, b}^{\mathrm{per}}$ with explicit error bounds.

\subsection{Floquet-Bloch transform}

Let $N$ be as in the statement of Theorem 3.3. Given a vector $x=\left(x_{n}\right)_{n \in \mathbb{Z}} \in \ell^{2}(\mathbb{Z})$ and given $\theta \in[0,2 \pi]$, define

$$
\left(\mathcal{U}_{\theta} x\right)_{n}:=(2 \pi)^{-\frac{1}{2}} \sum_{k \in \mathbb{Z}} x_{n+k N} e^{-1 \theta\left(k+\frac{n}{N}\right)} .
$$

We also introduce the symbol $\ell_{\text {per }}^{2}(N)$ to denote the space of all $N$-periodic sequences $\left(y_{k}\right)_{k \in \mathbb{Z}}$, together with the norm $\|y\|_{\ell_{\mathrm{per}}^{2}(N)}^{2}=\sum_{k=0}^{N-1}\left|y_{k}\right|^{2}$. Note that $\ell_{\mathrm{per}}^{2}(N)$ is canonically isomorphic to the Euclidean space $\mathbb{R}^{N}$. The following lemma is easily proved by direct computation.

Lemma 3.4 (Properties of $\mathcal{U}_{\theta}$ ) The $\operatorname{map} \mathcal{U}_{\theta}$ defined in (3.3) has the following properties.

(i) For any $x \in \ell^{2}(\mathbb{Z}), \mathcal{U}_{\theta} x$ is $N$-periodic, that is $\mathcal{U}_{\theta}: \ell^{2}(\mathbb{Z}) \rightarrow \ell_{\text {per }}^{2}(N)$;

(ii) The map

$$
\begin{aligned}
\mathcal{U}: \ell^{2}(\mathbb{Z}) & \rightarrow \int_{[0,2 \pi]}^{\oplus} \ell_{\mathrm{per}}^{2}(N) d \theta \\
x & \mapsto\left(\mathcal{U}_{\theta} x\right)_{\theta \in[0,2 \pi]}
\end{aligned}
$$

is unitary;

(iii) The inverse $\mathcal{U}^{-1}$ is given by

$$
\left(\mathcal{U}^{-1} y\right)_{n}=(2 \pi)^{-\frac{1}{2}} \int_{0}^{2 \pi} y_{n}(\theta) e^{1 n \frac{\theta}{N}} d \theta
$$

Proof This is standard, and the proof is omitted.

\subsection{Transform and properties of $A \in \Omega_{N, b}^{\text {per }}$}

The $N$-periodicity of $A \in \Omega_{N, b}^{\mathrm{per}}$ along diagonals is equivalent to the identity

$$
A_{m, n}=A_{m+k N, n+k N} \quad \text { for all } m, n, k \in \mathbb{Z} .
$$


Lemma 3.5 For any $A \in \Omega_{N, b}^{\mathrm{per}}, y \in \ell_{\mathrm{per}}^{2}(N)$ and $\theta \in[0,2 \pi]$, define $(A(\theta) y)_{n}=$ $\sum_{j \in \mathbb{Z}} e^{1 \theta \frac{j-n}{N}} A_{n j} y_{j}$. Then one has

$$
\mathcal{U} A \mathcal{U}^{-1}=\int_{[0,2 \pi]}^{\oplus} A(\theta) d \theta
$$

Proof A straightforward calculation (which we leave to the reader) shows that $\left(\mathcal{U}_{\theta} A x\right)_{n}=\left(A(\theta) \mathcal{U}_{\theta} x\right)_{n}$ for $n \in \mathbb{Z}$ and $x \in \ell^{2}(\mathbb{Z})$. The assertion now follows from the invertibility of $\mathcal{U}$.

Remark 3.6 Observe that $(A(\theta) y)_{n}=(A(\theta) y)_{n+k N}$ for any $k \in \mathbb{Z}$ so that $A(\theta) y \in$ $\ell_{\text {per }}^{2}(N)$. Hence, $A(\theta)$ is an operator $\ell_{\text {per }}^{2}(N) \rightarrow \ell_{\text {per }}^{2}(N)$, which can be expressed as an $N \times N$ matrix. Note, however, that the numbers $e^{-1 \theta \frac{n-j}{N}} A_{n j}$ are not the matrix elements of $A(\theta)$ with respect to any basis. Indeed, $\ell_{\text {per }}^{2}(N)$ is finite-dimensional, while $e^{-1 \theta \frac{n-j}{N}} A_{n j}(n, j \in \mathbb{Z})$ are infinitely many numbers.

As noted earlier, we have $\ell_{\text {per }}^{2}(N) \cong \mathbb{R}^{N}$. The identification can be made via the basis

$$
\begin{aligned}
& e_{1}^{\text {per }}=(\ldots, 0, \underbrace{1,0, \ldots, 0}_{N \text { entries }}, 1,0, \ldots) \\
& e_{2}^{\text {per }}=(\ldots, 0,0, \underbrace{1,0, \ldots, 0}_{N \text { entries }}, 1 \ldots)
\end{aligned}
$$

i.e. $\left(e_{n}^{\text {per }}\right)_{j}=\delta_{(j \bmod N), n}$ for $n \in\{0, \ldots, N-1\}$ (Kronecker symbol). In this basis, the matrix elements of $A(\theta)$ become

$$
\begin{aligned}
A(\theta)_{m n} & =\left\langle e_{m}^{\mathrm{per}}, A(\theta) e_{n}^{\mathrm{per}}\right\rangle_{\ell_{\mathrm{per}}^{2}(N)} \\
& =\sum_{k=0}^{N-1} \delta_{(k \bmod N), m} \sum_{j \in \mathbb{Z}} e^{1 \theta \frac{j-k}{N}} A_{k j} \delta_{(j \bmod N), n} \\
& =\sum_{j \in \mathbb{Z}} \sum_{k=0}^{N-1} \delta_{k, m} e^{1 \theta \frac{j-k}{N}} A_{k j} \delta_{(j \bmod N), n}
\end{aligned}
$$




$$
\begin{aligned}
& =\sum_{j \in \mathbb{Z}} e^{1 \theta \frac{j-m}{N}} A_{m j} \delta_{(j \bmod N), n} \\
& =\sum_{j^{\prime} \in \mathbb{Z}} e^{1 \theta \frac{j^{\prime} N+n-m}{N}} A_{m, j^{\prime} N+n} \\
& =e^{1 \theta \frac{n-m}{N}} \sum_{j^{\prime} \in \mathbb{Z}} e^{1 \theta j^{\prime}} A_{m, j^{\prime} N+n} .
\end{aligned}
$$

Note that the sum in the last line is actually finite, because $A$ is banded. Indeed, if the band width of $A$ is less than the period, then the sum over $j^{\prime}$ in (3.5) contains only one term.

Example 3.7 If $A$ is a matrix with $N=1$, i.e. $A$ is a Laurent operator, then formula (3.5) yields a scalar function of $\theta$ given by

$$
A(\theta)=\sum_{j \in \mathbb{Z}} e^{1 \theta j} A_{0, j}
$$

Writing $z:=e^{1 \theta}$, we see that $A(\theta)=\sum_{j \in \mathbb{Z}} z^{j} A_{0, j}$ is given by the symbol of the Laurent operator. We thus recover the classical result that the spectrum of a Laurent operator is given by the image of the unit circle under its symbol (cf. [39, Th. 7.1]).

Example 3.8 If $A$ is tri-diagonal and $N=5$ (cf. (3.2)), the formula above gives

$$
A(\theta)=\left(\begin{array}{ccccc}
a_{0} & b_{0} e^{1 \frac{\theta}{5}} & 0 & 0 & c_{0} e^{-1 \frac{\theta}{5}} \\
c_{1} e^{-1 \frac{\theta}{5}} & a_{1} & b_{1} e^{1 \frac{\theta}{5}} & 0 & 0 \\
0 & c_{2} e^{-1 \frac{\theta}{5}} & a_{2} & b_{2} e^{1 \frac{\theta}{5}} & 0 \\
0 & 0 & c_{3} e^{-1 \frac{\theta}{5}} & a_{3} & b_{3} e^{1 \frac{\theta}{5}} \\
b_{4} e^{1 \frac{\theta}{5}} & 0 & 0 & c_{4} e^{-1 \frac{\theta}{5}} & a_{4}
\end{array}\right) .
$$

Next, we establish some elementary facts about the spectrum of a periodic operator. By standard results about the Floquet-Bloch transform, we have

$$
\sigma(A)=\bigcup_{\theta \in[0,2 \pi]} \sigma(A(\theta))
$$

for all $A \in \Omega_{N, b}^{\mathrm{per}}$. Thus, an algorithm may be devised by determining the zeros of the map $z \mapsto \operatorname{det}(A(\theta)-z I), \theta \in[0,2 \pi]$. To this end, note that by definition of the determinant, one has

$$
\operatorname{det}(A(\theta)-z I)=\sum_{n=0}^{N} p_{n}(\theta) z^{n}
$$


where the coefficient functions $p_{n}(\theta)$ are polynomials in the matrix entries $A(\theta)_{m n}$ and hence analytic and periodic in $\theta$. Hence they are bounded:

$$
\exists C>0:\left|p_{n}(\theta)\right| \leq C \quad \forall \theta \in[0,2 \pi], \forall n \in\{0, \ldots, N\} .
$$

Moreover, $p_{N}(\theta) \equiv 1$.

Lemma 3.9 Let $A \in \Omega_{N, b}^{\mathrm{per}}$ and $R>0$. For any $z, w \in \mathbb{C}$ with $|z|,|w| \leq R$ and $\theta, \vartheta \in[0,2 \pi]$ one has

$$
\begin{aligned}
& |\operatorname{det}(A(\theta)-z I)-\operatorname{det}(A(\vartheta)-w I)| \\
& \quad \leq N^{\frac{N}{2}+2}\left((2 b+1)\|A\|_{\infty}+R\right)^{N}(2 b+1)^{2}\|A\|_{\infty}(|z-w|+|\theta-\vartheta|),
\end{aligned}
$$

where we note that $\|A\|_{\infty}=\max \left\{\left|A_{i j}\right| \mid i, j \in \mathbb{Z}\right\}$ can be computed in finitely many steps.

Proof Denote $B_{R}:=\{z \in \mathbb{C}|| z \mid \leq R\}$. From the mean value theorem it follows that for any differentiable function $f: B_{R} \times[0,2 \pi] \rightarrow \mathbb{C}$ one has

$$
|f(z, \theta)-f(w, \vartheta)| \leq\|\nabla f\|_{L^{\infty}\left(B_{R} \times[0,2 \pi]\right)}(|z-w|+|\theta-\vartheta|) .
$$

Hence to prove the claim it is enough to bound $\|\nabla f\|_{L^{\infty}\left(B_{R}\right) \times[0,2 \pi]}$ for $f(z, \theta)=$ $\operatorname{det}(A(\theta)-z I)$. This follows from the Jacobi formula: for any square matrix $M$ one has

$$
\frac{\partial \operatorname{det}(M)}{\partial M_{i j}}=\operatorname{cof}(M)_{i j},
$$

where $\operatorname{cof}(M)$ denotes the cofactor matrix of $M$. Hence,

$$
\begin{aligned}
\frac{\partial}{\partial z} \operatorname{det}(A(\theta)-z I) & =\sum_{i, j=0}^{N-1} \operatorname{cof}(A(\theta)-z I)_{i j}\left(-\delta_{i j}\right), \\
\frac{\partial}{\partial \theta} \operatorname{det}(A(\theta)-z I) & =\sum_{i, j=0}^{N-1} \operatorname{cof}(A(\theta)-z I)_{i j} \frac{\partial A(\theta)_{i j}}{\partial \theta} .
\end{aligned}
$$

Using Hadamard's inequality to bound the cofactor matrix, we obtain the bounds

$$
\begin{aligned}
\left|\frac{\partial}{\partial z} \operatorname{det}(A(\theta)-z I)\right| & \leq N^{\frac{N}{2}+1}\|A(\theta)-z I\|_{\infty}^{N} \\
& \leq N^{\frac{N}{2}+1}\left((2 b+1)\|A\|_{\infty}+R\right)^{N} \\
\left|\frac{\partial}{\partial \theta} \operatorname{det}(A(\theta)-z I)\right| & \leq N^{\frac{N}{2}+2}\|A(\theta)-z I\|_{\infty}^{N}\left\|\partial_{\theta} A(\theta)\right\|_{\infty} \\
& \leq N^{\frac{N}{2}+2}\left((2 b+1)\|A\|_{\infty}+R\right)^{N}\left\|\partial_{\theta} A(\theta)\right\|_{\infty} \\
& \leq N^{\frac{N}{2}+2}\left((2 b+1)\|A\|_{\infty}+R\right)^{N}(2 b+1)^{2}\|A\|_{\infty},
\end{aligned}
$$


where the last two lines follow from the explicit formula (3.5). The bounds (3.6) and (3.7) imply

$$
\max \left\{\left|\partial_{\theta} f\right|,\left|\partial_{z} f\right|\right\} \leq N^{\frac{N}{2}+2}\left((2 b+1)\|A\|_{\infty}+R\right)^{N}(2 b+1)^{2}\|A\|_{\infty}
$$

and the claim follows.

\subsection{Proof of Theorem 1.1(ii)}

We can finally prove Theorem 1.1(ii) which was restated equivalently as Theorem 3.3. First, we define the family of algorithms $\left\{\Gamma_{n}\right\}_{n \in \mathbb{N}}$, where each of them maps $\Gamma_{n}: \Omega_{N, b}^{\text {per }} \rightarrow \mathcal{M}$ (we recall that $\mathcal{M}$ is the space of all compact subsets of $\mathbb{C}$ endowed with the Hausdorff metric). It is easy to see that for any $A \in \Omega_{N, b}^{\text {per }}$ one has $\|A\|_{\ell^{2} \rightarrow \ell^{2}} \leq$ $R_{A}:=\sum_{j=1}^{N} \sum_{k=-b}^{b}\left|A_{j k}\right|$ (this follows from Young's inequality), a quantity which can be computed in finitely many steps. Therefore, if we denote $B_{R_{A}}:=\{z \in \mathbb{C}|| z \mid \leq$ $\left.R_{A}\right\}$, the a priori inclusion $\sigma(A) \subset B_{R_{A}}$ holds true for any $A \in \Omega_{N, b}^{\mathrm{per}}$.

Definition 3.10 ( $N$, b-Periodic Matrix Algorithm) Let $A \in \Omega_{N, b}^{\mathrm{per}}$ and for $n \in \mathbb{N}$, let $\Theta_{n}=\left(\theta_{1}^{(n)}, \ldots, \theta_{n}^{(n)}\right)$ be a linear spacing of $[0,2 \pi]$ and let $L_{n}:=\frac{1}{n}(\mathbb{Z}+1 \mathbb{Z}) \cap B_{R_{A}}$ be a finite lattice with spacing $n^{-1}$. Then we define

$$
\Gamma_{n}(A):=\bigcup_{i=1}^{n}\left\{z \in L_{n}|| \operatorname{det}\left(A\left(\theta_{i}^{(n)}\right)-z I\right) \mid \leq n^{-\frac{1}{2}}\right\} .
$$

Remark 3.11 We emphasize that (3.8) can be computed in finitely many arithmetic operations on the matrix elements of $A$. Indeed, computing the radius $R_{A}$ consists of a finite sequence of multiplications and additions, as does the computation of each of the determinants $\operatorname{det}\left(A\left(\theta_{i}^{(n)}\right)-z\right)$ for $z, \theta_{i}^{(n)}$ in the finite sets $L_{n}, \Theta_{n}$.

Proof of Theorem 3.3 (equiv. Theorem 1.1(ii)) The proof has two steps.

Step 1: $\sigma(A)$ is approximated from above by $\Gamma_{n}(A)$. For any set $K \subset \mathbb{C}$ we denote by $B_{\delta}(K)$ the $\delta$-neighborhood of $K$.

Let $z \in \sigma(A)$. Then $|z| \leq\|A\|_{\ell^{2} \rightarrow \ell^{2}} \leq R_{A}$ and there exists $\theta \in[0,2 \pi]$ such that $\operatorname{det}(A(\theta)-z I)=0$. Choose $z_{n} \in L_{n}$ such that $\left|z-z_{n}\right| \leq n^{-1}$ and $\theta_{n} \in \Theta_{n}$ such that $\left|\theta-\theta_{n}\right| \leq n^{-1}$. Applying Lemma 3.9 we obtain the bound

$$
\begin{aligned}
\left|\operatorname{det}\left(A\left(\theta_{n}\right)-z_{n} I\right)\right| \leq & N^{\frac{N}{2}+2}\left((2 b+1)\|A\|_{\infty}+R_{A}\right)^{N}(2 b+1)^{2}\|A\|_{\infty} \\
& \times\left(\left|z-z_{n}\right|+\left|\theta-\theta_{n}\right|\right) \\
& \leq \frac{2}{n} N^{\frac{N}{2}+2}\left((2 b+1)\|A\|_{\infty}+R_{A}\right)^{N}(2 b+1)^{2}\|A\|_{\infty} .
\end{aligned}
$$


This inequality implies that $z_{n} \in \Gamma_{n}(A)$ as soon as $2 n^{-1} N^{\frac{N}{2}+2}\left((2 b+1)\|A\|_{\infty}+\right.$ $\left.R_{A}\right)^{N}(2 b+1)^{2}\|A\|_{\infty} \leq n^{-\frac{1}{2}}$, or equivalently,

$$
n>\left(2 N^{\frac{N}{2}+2}\left((2 b+1)\|A\|_{\infty}+R_{A}\right)^{N}(2 b+1)^{2}\|A\|_{\infty}\right)^{2} .
$$

Note that the right-hand side of (3.9) is computable in finitely many arithmetic operations if $N$ and $b$ are known a priori. Since $\left|z-z_{n}\right| \leq n^{-1}$ by construction, this shows that $\sigma(A) \subset B_{\frac{1}{n}}\left(\Gamma_{n}(A)\right)$ for $n>\left(2 N^{\frac{N}{2}+2}\left((2 b+1)\|A\|_{\infty}+R_{A}\right)^{N}(2 b+1)^{2}\|A\|_{\infty}\right)^{2}$.

Step 2: $\sigma(A)$ is approximated from below by $\Gamma_{n}(A)$. Next we prove that $\Gamma_{n}(A) \subset$

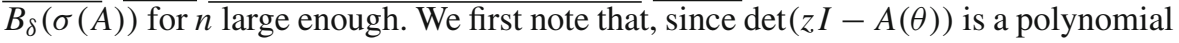
in $z$, it can be factored to take the form

$$
\operatorname{det}(z I-A(\theta))=\prod_{i=1}^{N}\left(z-z_{i}(\theta)\right)
$$

where $z_{i}(\theta)$ are the zeros of $z \mapsto \operatorname{det}(z I-A(\theta)$ ) (note that for a characteristic polynomial the coefficient of the leading order term is always 1). From (3.10) we obtain the bound

$$
|\operatorname{det}(z I-A(\theta))|=\prod_{i=1}^{N}\left|z-z_{i}(\theta)\right| \geq \operatorname{dist}(z, \sigma(A))^{N} .
$$

Let $z_{n} \in \Gamma_{n}(A)$ be an arbitrary sequence. Then, by definition, $\left|\operatorname{det}\left(z_{n} I-A\left(\theta_{n}\right)\right)\right| \leq$ $n^{-\frac{1}{2}}$ for some $\theta_{n} \in \Theta_{n}$. From (3.11) we conclude that

$$
\operatorname{dist}\left(z_{n}, \sigma(A)\right)^{N} \leq\left|\operatorname{det}\left(z_{n} I-A\left(\theta_{n}\right)\right)\right| \leq n^{-\frac{1}{2}}
$$

and thus $z_{n} \in B_{n^{-1 / 2 N}}(\sigma(A))$ for all $n \in \mathbb{N}$. This concludes step 2 .

Together, steps 1 and 2 imply that for any given $\delta>0$ one has both $\Gamma_{n}(A) \subset$ $B_{\delta}(\sigma(A))$ and $\sigma(A) \subset B_{\delta}\left(\Gamma_{n}(A)\right)$ provided that

$$
n>\max \left\{\delta^{-2 N},\left(2 N^{\frac{N}{2}+2}\left((2 b+1)\|A\|_{\infty}+R_{A}\right)^{N}(2 b+1)^{2}\|A\|_{\infty}\right)^{2}\right\} .
$$

Since the right-hand side of (3.12) is computable from $N, b$ and the matrix elements of $A$ in finitely many arithmetic operations, we conclude that the computational problem is in $\Delta_{1}$.

Example 3.12 The algorithm from Definition 3.10 can easily be implemented in Matlab. An example calculation with bandwidth $b=1, N=5$ and $A$ of the form (3.2) with 

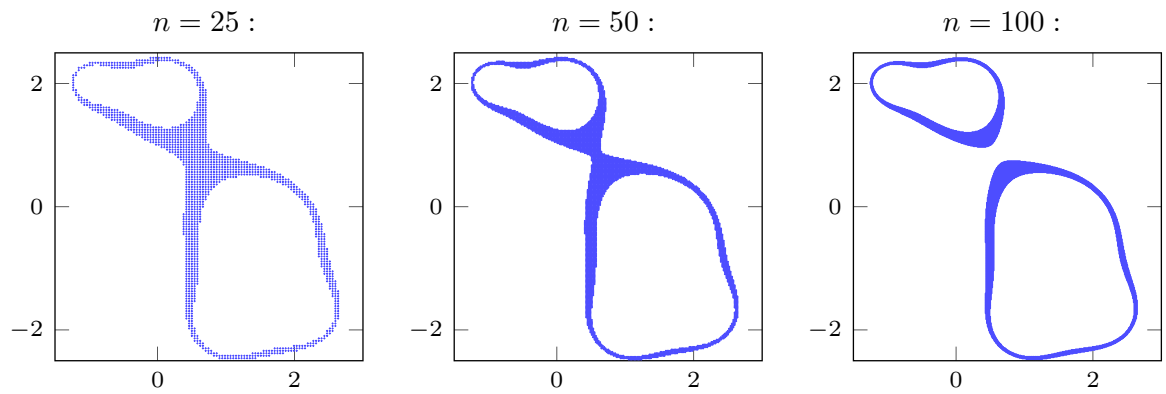

Fig. 2 Output of $\Gamma_{n}(A)$ in the complex plane for different values of $n$

$$
\begin{aligned}
& \left(a_{i}\right)=(1,0,1,0,2) \\
& \left(b_{i}\right)=(-1,-2,1,31,-5) \\
& \left(c_{i}\right)=(21,-31,21,0,1)
\end{aligned}
$$

yields the following output in the complex plane. Note that the output is a set of points on a discrete grid in the complex plane and hence the output looks 'fat'. As $n$ is taken larger this set of points dwindles to just those points that lie in an ever decreasing neighborhood of the true spectrum.

The Matlab implementation that produced Fig. 2 is available online at https://github. com/frank-roesler/TriSpec.

\section{Schrödinger operators with periodic potentials}

In this section we prove Theorem 1.2 regarding the spectrum of Schrödinger operators $H=-\Delta+V$ with periodic potentials $V: \mathbb{R}^{d} \rightarrow \mathbb{C}$. Again, this is done by defining an explicit algorithm. We shall consider three computational problems which only differ in their primary set $\Omega$, and are as follows (primary sets are defined immediately below):

$$
\left\{\begin{aligned}
\Omega & =\Omega^{\mathrm{Sch}} \text { or } \Omega_{p}^{\mathrm{Sch}} \text { or } \Omega_{p, M}^{\mathrm{Sch}} \\
\mathcal{M} & =\left(\{K \subset \mathbb{C} \mid K \text { closed }\}, d_{\mathrm{AW}}\right) \\
\Lambda & =\left\{V \mapsto V(x) \mid x \in \mathbb{R}^{d}\right\} \\
\Xi: & \Omega \rightarrow \mathcal{M} ; \quad V \mapsto \sigma(-\Delta+V),
\end{aligned}\right.
$$

where $-\Delta+V$ is meant to be defined on $L^{2}\left(\mathbb{R}^{d}\right)$ with domain $H^{2}\left(\mathbb{R}^{d}\right)$, and $d_{\text {AW }}$ denotes the Attouch-Wets metric, which is a generalization of the Hausdorff metric for the case of sets which may be unbounded (see Appendix A for a brief discussion). Note that the spectrum $\sigma(-\Delta+V)$ is always non-empty in this case, so taking this metric makes sense. For $p>d$ and $M>0$, the primary sets are defined as follows: 


$$
\begin{aligned}
\Omega_{p}^{\mathrm{Sch}} & :=\left\{V: \mathbb{R}^{d} \rightarrow \mathbb{C} \mid V \text { is 1-periodic and }\left.V\right|_{(0,1)^{d}} \in W^{1, p}\left((0,1)^{d}\right)\right\} \\
\Omega^{\mathrm{Sch}} & :=\bigcup_{p>d} \Omega_{p}^{\mathrm{Sch}} \\
\Omega_{p, M}^{\mathrm{Sch}} & :=\left\{V \in \Omega_{p}^{\mathrm{Sch}} \mid\|V\|_{W^{1, p}\left((0,1)^{d}\right)} \leq M\right\}
\end{aligned}
$$

Note that by Morrey's inequality, every $V \in \Omega_{p}^{\text {Sch }}$ is continuous, and so the evaluation set $\Lambda$ which comprises point evaluations of $V$, is well-defined. In the language of the Solvability Complexity Index, the three parts of Theorem 1.2 can be expressed as follows:

- Part (i) amounts to proving that the computational problem for $\Omega^{\text {Sch }}$ has an SCI value of 1 (or, equivalently, it belongs to $\Delta_{2}$ ).

- Part (ii) amounts to showing that the computational problem for $\Omega_{p, M}^{\mathrm{Sch}}$ belongs to $\Pi_{1}$, i.e. it can be approximated from above with explicit error bounds.

- Part (iii) amounts to showing that the computational problem for potentials that are real-valued and in $\Omega_{p, M}^{\mathrm{Sch}}$ belongs to $\Delta_{1}$, i.e. it can be approximated with explicit error bounds.

The proof of Theorem 1.2 is contained in Subsection 4.3, and it relies on the following weaker theorem:

Theorem 4.1 The computational problem for $\Omega_{p}^{\mathrm{Sch}}$ can be solved in one limit: $\operatorname{SCI}\left(\Omega_{p}^{\mathrm{Sch}}\right)=1$ (equivalently, $\Omega_{p}^{\mathrm{Sch}} \in \Delta_{2}$ ).

Note that this theorem is evidently weaker than Theorem 1.2(i) as the class of potentials $\Omega_{p}^{\mathrm{Sch}}$ considered here is a strict subset of the class $\Omega^{\mathrm{Sch}}=\cup_{p>d} \Omega_{p}^{\mathrm{Sch}}$ considered in Theorem 1.2(i). The proof is constructive, i.e. we provide an explicit algorithm that computes the spectrum of any given operator with $V \in \Omega_{p}^{\text {Sch }}$. Note that this problem is fundamentally different from the discrete problem (3.1) where we could directly access the matrix elements of the (discrete) operator. Instead, the evaluation set $\Lambda$ gives access to the point values of the potential. Hence our task is to construct a sequence of algorithms $\left\{\Gamma_{n}\right\}_{n \in \mathbb{N}}$, such that each $\Gamma_{n}$ computes its output from finitely many point evaluations of $V$ using finitely many algebraic operations.

The proof of Theorem 4.1 is contained in Subsection 4.2. Prior to that, Subsection 4.1 contains several technical estimates needed to prove convergence of the algorithm.

\subsection{Estimating and approximating the potential}

This section uses well-known facts about the Floquet-Bloch transform, which we gather in Appendix B. The critical step is to compute approximations to the spectrum $\sigma(-\Delta+V)$ using only finitely many pointwise evaluations of $V$. This is done using the Floquet-Bloch transform in conjunction with the Birman-Schwinger principle. The approximate potential is defined in (4.8) and the critical error bound is stated in Lemma 4.6. 


\subsubsection{A Birman-Schwinger principle for $H(\theta)$}

It shall be convenient for us to characterize the eigenvalues using the well-known Birman-Schwinger principle [8,33]. This principle requires a decomposition, which we choose as follows. Let $p>d, V \in \Omega_{p}^{\mathrm{Sch}}, \theta \in[0,2 \pi]^{d}$ and let $H(\theta)$ be the corresponding Floquet-Bloch operator as in (B.1). Expanding the operator square, $H(\theta)$ can be written as

$$
H(\theta)=-\Delta-21 \theta \cdot \nabla+|\theta|^{2}+V
$$

Let us choose the following decomposition of $H(\theta)$. We define

$$
\begin{aligned}
H_{0} & :=-\Delta+1, & \operatorname{dom}\left(H_{0}\right) & =H_{\mathrm{per}}^{2}\left((0,1)^{d}\right), \\
B(\theta) & :=-21 \theta \cdot \nabla+|\theta|^{2}-1+V, & \operatorname{dom}(B(\theta)) & =H_{\mathrm{per}}^{1}\left((0,1)^{d}\right) .
\end{aligned}
$$

Next we derive an operator identity that characterizes $\lambda \in \sigma(H(\theta)) \backslash \sigma\left(H_{0}\right)$ as those points for which $1 \in \sigma(\mathcal{K}(\lambda)$ ), for a certain compact operator $\mathcal{K}(\lambda)$. Clearly one has $H(\theta)=H_{0}+B(\theta)$. The auxiliary constant 1 , which is added in $H_{0}$ and subtracted again in $B(\theta)$ was chosen for convenience, so that $H_{0}$ becomes a positive invertible operator. Note that $B(\theta)$ is relatively compact with respect to $H_{0}$. For $\lambda \notin \sigma\left(H_{0}\right)$ one has

$$
\lambda-H_{0}-B(\theta)=H_{0}^{\frac{1}{2}}\left(I-H_{0}^{-\frac{1}{2}} B(\theta)\left(\lambda-H_{0}\right)^{-1} H_{0}^{\frac{1}{2}}\right) H_{0}^{-\frac{1}{2}}\left(\lambda-H_{0}\right),
$$

where $I$ denotes the identity operator on $L^{2}\left((0,1)^{d}\right)$ It follows that $\lambda-H_{0}-B(\theta)$ is invertible if and only if $I-H_{0}^{-1 / 2} B(\theta)\left(\lambda-H_{0}\right)^{-1} H_{0}^{1 / 2}$ is invertible, and in that case

$$
\left(\lambda-H_{0}-B(\theta)\right)^{-1}=\left(\lambda-H_{0}\right)^{-1} H_{0}^{\frac{1}{2}}\left(I-H_{0}^{-\frac{1}{2}} B(\theta) H_{0}^{\frac{1}{2}}\left(\lambda-H_{0}\right)^{-1}\right)^{-1} H_{0}^{-\frac{1}{2}} .
$$

This identity implies that

$$
\lambda \in \mathbb{C} \backslash \sigma\left(H_{0}\right) \text { is in } \sigma(H(\theta)) \Leftrightarrow 1 \in \sigma\left(H_{0}^{-\frac{1}{2}} B(\theta) H_{0}^{\frac{1}{2}}\left(\lambda-H_{0}\right)^{-1}\right) .
$$

\subsubsection{Schatten class estimates}

We now study the analytic operator valued function

$$
K(\lambda, \theta):=H_{0}^{-\frac{1}{2}} B(\theta) H_{0}^{\frac{1}{2}}\left(\lambda-H_{0}\right)^{-1} .
$$

We choose a Fourier basis for $L^{2}\left((0,1)^{d}\right)$, that is, we choose a numbering $\mathbb{N} \ni j \mapsto$ $k_{j} \in 2 \pi \mathbb{Z}^{d}$ such that $\left|k_{j}\right|$ is monotonically increasing with $j$ and set

$$
e_{j}:=e^{1 k_{j} \cdot x}
$$


We note that $e_{j} \in \operatorname{dom}(H(\theta))$ for all $\theta$ and $\left\|e_{j}\right\|_{L^{2}\left((0,1)^{d}\right)}=1$ for all $j \in \mathbb{N}$. In this basis, the operators $H_{0}^{1 / 2}, H_{0}$ and $\theta \cdot \nabla$ are all diagonal and one has

$$
\begin{aligned}
H_{0}^{1 / 2} & =\operatorname{diag}\left(\left(1+\left|k_{j}\right|^{2}\right)^{1 / 2}\right) \\
\lambda-H_{0} & =\operatorname{diag}\left(\lambda-1-\left|k_{j}\right|^{2}\right) \\
-21 \theta \cdot \nabla & =\operatorname{diag}\left(2 \theta \cdot k_{j}\right) .
\end{aligned}
$$

Therefore, we have

$$
H_{0}^{-\frac{1}{2}}(-21 \theta \cdot \nabla) H_{0}^{\frac{1}{2}}\left(\lambda-H_{0}\right)^{-1}=\operatorname{diag}\left(\frac{2 \theta \cdot k_{j}}{\lambda-1-\left|k_{j}\right|^{2}}\right) .
$$

Now the following lemma is easily proved.

Lemma 4.2 (Schatten bound for $K$ ) For every $s>d$ the operator $K(\lambda, \theta)$ belongs to the Schatten class $\mathcal{C}_{s}$ and one has

$$
\|K(\lambda, \theta)\|_{\mathcal{C}_{s}} \leq\left(\frac{2}{\pi}|\theta|+\frac{2}{\pi}\left\||\theta|^{2}-1+V\right\|\right) C_{\lambda}\left(1-\frac{d}{s}\right)^{-\frac{1}{s}}
$$

where $C_{\lambda}:=\sup _{j \in \mathbb{N}}\left|1-\frac{\lambda-1}{\left|k_{j}\right|^{2}}\right|^{-1}$ and $\|\cdot\|$ denotes the $L^{2}$ operator norm.

Proof Let $\lambda \in \mathbb{C} \backslash \sigma\left(H_{0}\right)$ and note that $C_{\lambda}<+\infty$ by our choice of $\lambda$. Observe that simple geometric considerations lead to the bound

$$
\left|k_{j}\right| \geq \pi j^{\frac{1}{d}}
$$

Then one has

$$
\begin{aligned}
\left|\frac{2 \theta \cdot k_{j}}{\lambda-1-\left|k_{j}\right|^{2}}\right| & \leq 2 \frac{|\theta|\left|k_{j}\right|}{\left.|\lambda-1-| k_{j}\right|^{2} \mid} \\
& \leq 2 C_{\lambda}|\theta|\left|k_{j}\right|^{-1} \\
& \leq \frac{2}{\pi} C_{\lambda}|\theta| j^{-\frac{1}{d}} .
\end{aligned}
$$

Hence the characteristic numbers of $H_{0}^{-\frac{1}{2}}(-21 \theta \cdot \nabla) H_{0}^{\frac{1}{2}}\left(\lambda-H_{0}\right)^{-1}$ are bounded by $\frac{2}{\pi} C_{\lambda}|\theta| j^{-\frac{1}{d}}$ and thus one has

$$
H_{0}^{-\frac{1}{2}}(-21 \theta \cdot \nabla) H_{0}^{\frac{1}{2}}\left(\lambda-H_{0}\right)^{-1} \in \mathcal{C}_{s} \quad \text { for any } \quad s>d
$$


and

$$
\begin{aligned}
\left\|H_{0}^{-\frac{1}{2}}(-21 \theta \cdot \nabla) H_{0}^{\frac{1}{2}}\left(\lambda-H_{0}\right)^{-1}\right\|_{\mathcal{C}_{s}} & \leq \frac{2}{\pi} C_{\lambda}|\theta|\left(\sum_{j=1}^{\infty} j^{-\frac{s}{d}}\right)^{\frac{1}{s}} \\
& \leq \frac{2}{\pi} C_{\lambda}|\theta|\left(1-\frac{d}{s}\right)^{-\frac{1}{s}} .
\end{aligned}
$$

Next we turn to the potential term in $K(\lambda, \theta)$, that is, the operator $H_{0}^{-1 / 2}\left(|\theta|^{2}-1+\right.$ $V) H_{0}^{1 / 2}\left(\lambda-H_{0}\right)^{-1}$. This is easily treated by the ideal property of $\mathcal{C}_{s}$, since the operator $|\theta|^{2}-1+V$ is bounded. Indeed, we have for every $s>d$

$$
\begin{aligned}
& \left\|H_{0}^{-1 / 2}\left(|\theta|^{2}-1+V\right) H_{0}^{1 / 2}\left(\lambda-H_{0}\right)^{-1}\right\|_{\mathcal{C}_{s}} \\
& \quad \leq\left\|H_{0}^{-1 / 2}\right\|\left\||\theta|^{2}-1+V\right\|\left\|H_{0}^{1 / 2}\left(\lambda-H_{0}\right)^{-1}\right\|_{\mathcal{C}_{s}} \\
& \quad \leq\left\||\theta|^{2}-1+V\right\| \frac{2}{\pi} C_{\lambda}\left(1-\frac{d}{s}\right)^{-\frac{1}{s}}
\end{aligned}
$$

where the last line follows from a similar calculation to (4.4) and the fact that $\left\|H_{0}^{-1 / 2}\right\|=1$ (this follows from the matrix representation (4.2) and the fact that $\left.k_{1}=0\right)$.

Lemma 4.3 (Lipschitz continuity of $K$ ) For every $s>d$ and $\lambda, \mu \in \mathbb{C} \backslash \sigma\left(H_{0}\right)$, $\theta, \vartheta \in[0,2 \pi]^{d}$ one has

$$
\|K(\lambda, \theta)-K(\mu, \vartheta)\|_{\mathcal{C}_{s}} \leq 8 d^{\frac{1}{2}} C_{\lambda}\left(1-\frac{d}{s}\right)^{-\frac{1}{s}}\left(|\theta-\vartheta|+c_{V} C_{\mu}|\lambda-\mu|\right),
$$

where $C_{\lambda}$ is defined as in Lemma 4.2 and $c_{V}=\frac{1}{2}+\pi d^{\frac{1}{2}}+\left(4 \pi d^{\frac{1}{2}}\right)^{-1}\|V-1\|_{\infty}$.

Proof First, note that

$$
B(\theta)-B(\vartheta)=-21(\theta-\vartheta) \cdot \nabla+(\theta+\vartheta) \cdot(\theta-\vartheta)
$$

Using (4.7) together with the resolvent identity for $\left(\lambda-H_{0}\right)^{-1}$ one obtains

$$
\begin{aligned}
K(\lambda, \theta)-K(\mu, \vartheta)= & H_{0}^{-\frac{1}{2}}(-21(\theta-\vartheta) \cdot \nabla+(\theta+\vartheta) \cdot(\theta-\vartheta)) H_{0}^{\frac{1}{2}}\left(\lambda-H_{0}\right)^{-1} \\
& +H_{0}^{-\frac{1}{2}} B(\vartheta) H_{0}^{\frac{1}{2}}(\lambda-\mu)\left(\lambda-H_{0}\right)^{-1}\left(\mu-H_{0}\right)^{-1} \\
= & (\theta-\vartheta) \cdot(-21 \nabla+\theta+\vartheta)\left(\lambda-H_{0}\right)^{-1} \\
& +(\lambda-\mu) K(\lambda, \vartheta)\left(\mu-H_{0}\right)^{-1}
\end{aligned}
$$


Taking norms in $\mathcal{C}_{s}$ and using the estimates from the proof of Lemma 4.2 (and in particular (4.5)) we obtain the bound

$$
\begin{aligned}
\|K(\lambda, \theta)-K(\mu, \vartheta)\|_{\mathcal{C}_{s} \leq} & |\theta-\vartheta|\left\|(-21 \nabla+\theta+\vartheta)\left(\lambda-H_{0}\right)^{-1}\right\|_{\mathcal{C}_{s}} \\
& +|\lambda-\mu|\|K(\lambda, \vartheta)\|_{\mathcal{C}_{s}}\left\|\left(\mu-H_{0}\right)^{-1}\right\| \\
\leq & |\theta-\vartheta| \frac{2}{\pi} C_{\lambda}|\theta+\vartheta|\left(1-\frac{d}{s}\right)^{-\frac{1}{s}} \\
& +|\lambda-\mu|\|K(\lambda, \vartheta)\|_{\mathcal{C}_{s}} C_{\mu} .
\end{aligned}
$$

Finally, applying Lemma 4.2 and using $|\theta+\vartheta| \leq 4 \pi d^{\frac{1}{2}}$ we obtain

$$
\begin{aligned}
\|K(\lambda, \theta)-K(\mu, \vartheta)\|_{\mathcal{C}_{s} \leq} & |\theta-\vartheta| 8 C_{\lambda} d^{\frac{1}{2}}\left(1-\frac{d}{s}\right)^{-\frac{1}{s}} \\
& +|\lambda-\mu|\left(4 d^{\frac{1}{2}}+8 \pi d+\frac{2}{\pi}\|V-1\|\right) C_{\lambda} C_{\mu}\left(1-\frac{d}{s}\right)^{-\frac{1}{s}} \\
\leq & 8 d^{\frac{1}{2}} C_{\lambda}\left(1-\frac{d}{s}\right)^{-\frac{1}{s}} \\
& \left(|\theta-\vartheta|+\left(\frac{1}{2}+\pi d^{\frac{1}{2}}+\left(4 \pi d^{\frac{1}{2}}\right)^{-1}\|V-1\|\right) C_{\mu}|\lambda-\mu|\right) .
\end{aligned}
$$

This concludes the proof.

While Lemma 4.3 gives precise information about the dependence of the Lipschitz constant of $K$ on all parameters, it will be useful for us to have a bound which is less precise but more explicit (and manifestly computable).

Corollary 4.4 Let $s>d$ and $\delta, R>0$. Then for any $\theta, \vartheta \in[0,2 \pi]^{d}$ and $\lambda, \mu \in \mathbb{C}$ such that $\left|\lambda-\left(1+\left|k_{j}\right|^{2}\right)\right|,\left|\mu-\left(1+\left|k_{j}\right|^{2}\right)\right|>\delta$ for all $j$ and $|\lambda|,|\mu|<R$ one has

$$
\begin{aligned}
\|K(\lambda, \theta)-K(\mu, \vartheta)\|_{\mathcal{C}_{s} \leq} \leq & \delta^{-2} 48 R^{2} \frac{s d}{s-d} \frac{3 p-d}{p-d} \times \\
& \left(1+\|V\|_{W^{1, p}\left((0,1)^{d}\right)}\right)(|\theta-\vartheta|+|\lambda-\mu|),
\end{aligned}
$$

Proof This follows immediately from Lemma 4.3 via rather crude estimates, noting that $C_{\lambda} \leq R \delta^{-1}$ and $\|V\|_{\infty} \leq \frac{3 p-d}{p-d}\|V\|_{W^{1, p}\left((0,1)^{d}\right)}$ (cf. [9, Ch. 9.3]).

\subsubsection{Approximation of the potential}

Next we study the matrix representation of the potential $V$ in the Fourier basis $\left\{e_{j}\right\}_{j \in \mathbb{N}}$ and its approximations. First, we observe that in the Fourier basis $\left\{e_{j}\right\}_{j \in \mathbb{N}}$ one has

$$
\left\langle e_{j}, V e_{m}\right\rangle=\hat{V}_{k_{m}-k_{j}}
$$


where $\hat{V}_{k}$ denote the Fourier coefficients of $V$. Indeed, a direct calculation gives

$$
\left\langle e_{j}, V e_{m}\right\rangle=\int_{(0,1)^{d}} \overline{e^{1 k_{j} \cdot x}} V(x) e^{1 k_{m} \cdot x} d x=\int_{(0,1)^{d}} V(x) e^{1\left(k_{m}-k_{j}\right) \cdot x} d x=\hat{V}_{k_{m}-k_{j}}
$$

Now, we want to build a computable, finite size approximation of the matrix $\left(V_{j m}\right)=$ $\left(\left\langle e_{j}, V e_{m}\right\rangle\right)$. We start by approximating the Fourier coefficients $\hat{V}_{k}$.

Lemma 4.5 Let $n \in \mathbb{N}$ and define the lattice $I_{n}:=\left\{\frac{m}{n} \mid m=0, \ldots, n-1\right\}^{d} \subset(0,1)^{d}$. For every $f \in W^{1, p}\left((0,1)^{d}\right), p>d$, one has

$$
\left|n^{-d} \sum_{\xi \in I_{n}} f(\xi)-\int_{(0,1)^{d}} f(x) d x\right| \leq \frac{2 n^{-1+d / p}}{1-d / p}\|\nabla f\|_{L^{p}\left((0,1)^{d}\right)}
$$

Proof The proof is a standard application of Morrey's inequality (cf. (28) in the proof of [9, Th. 9.12]), which implies that $|f(x)-f(\xi)| \leq \frac{2 n^{-1+d / p}}{1-d / p}\|\nabla f\|_{L^{p}\left((0,1)^{d}\right)}$ for $x$ in the cube $\left(0, \frac{1}{n}\right)^{d}+\xi$. Details are left to the reader.

Let us introduce the approximate Fourier coefficients for $k \in 2 \pi \mathbb{Z}^{d}$ and $n \in \mathbb{N}$,

$$
\hat{V}_{k}^{\mathrm{appr}, n}:=n^{-d} \sum_{\xi \in I_{n}} V(\xi) e^{1 k \cdot \xi}
$$

Note that the $\hat{V}_{k}^{\text {appr, } n}$ can be computed in finitely many operations from the information provided in $\Lambda$. Lemma 4.5 applied to the function $f(x)=V(x) e^{1 k \cdot x}$ leads to the error estimate

$$
\begin{aligned}
\left|\hat{V}_{k}^{\mathrm{appr}, n}-\hat{V}_{k}\right| & \leq \frac{2 n^{-1+d / p}}{1-d / p}\left\|\nabla\left(V e^{1 k \cdot x}\right)\right\|_{L^{p}\left((0,1)^{d}\right)} \\
& \leq \frac{2 n^{-1+d / p}}{1-d / p}\left(\left\|\nabla V e^{1 k \cdot x}\right\|_{L^{p}\left((0,1)^{d}\right)}+\left\|V \nabla e^{1 k \cdot x}\right\|_{L^{p}\left((0,1)^{d}\right)}\right) \\
& =\frac{2 n^{-1+d / p}}{1-d / p}\left(\|\nabla V\|_{L^{p}\left((0,1)^{d}\right)}+|k|\|V\|_{L^{p}\left((0,1)^{d}\right)}\right) \\
& \leq \frac{2 n^{-1+d / p}}{1-d / p}(1+|k|)\|V\|_{W^{1, p}\left((0,1)^{d}\right)}
\end{aligned}
$$

Next, we define the approximate potential matrix

$$
V^{\mathrm{appr}, n}:=\left(\hat{V}_{k_{m}-k_{j}}^{\mathrm{appr}, n}\right)_{m, j \in \mathbb{N}} \cdot
$$

We remark that the approximated potential matrix cannot be computed in finitely many algebraic operations from the point values of $V$, because it has infinitely many entries. This issue will be addressed next. 
Lemma 4.6 (Main Error Bound) For $N \in \mathbb{N}$ let $\mathcal{H}_{N}=\operatorname{Span}\left\{e_{1}, \ldots, e_{N}\right\}$ and let $P_{N}: L^{2}\left((0,1)^{d}\right) \rightarrow \mathcal{H}_{N}$ be the orthogonal projection. Moreover, define

$$
K_{n}^{\text {appr }}(\lambda, \theta):=H_{0}^{-\frac{1}{2}}\left(-21 \theta \cdot \nabla+|\theta|^{2}-1+V^{\text {appr }, n}\right) H_{0}^{\frac{1}{2}}\left(\lambda-H_{0}\right)^{-1} .
$$

Then for every $s>d$ one has

$$
\begin{aligned}
& \left\|K(\lambda, \theta)-P_{N} K_{n}^{\mathrm{appr}}(\lambda, \theta) P_{N}\right\|_{\mathcal{C}_{s}} \\
& \leq C_{\lambda}\left(C_{s, d}^{1}|\theta| N^{\frac{1}{s}-\frac{1}{d}}+C_{s, p, d}^{2} \frac{N^{1+\frac{1}{d}}}{n^{1-\frac{d}{p}}}\|V\|_{W^{1, p}\left((0,1)^{d}\right)}\right. \\
& \left.\quad+C_{s, d}^{3} N^{\frac{1}{s}-\frac{1}{d}}\left(\left.|| \theta\right|^{2}-1 \mid+\|V\|_{L^{\infty}\left((0,1)^{d}\right)}\right)\right),
\end{aligned}
$$

where we recall that $C_{\lambda}=\sup _{j \in \mathbb{N}}\left|1-\frac{\lambda-1}{\left|k_{j}\right|^{2}}\right|^{-1}$ and $C_{s, d}^{1}, C_{s, p, d}^{2}, C_{s, d}^{3}$ are explicit constants independent of $n, N, \lambda, \theta$.

Proof Again, we denote by $\|\cdot\|$ the $L^{2}\left(\mathbb{R}^{d}\right)$ operator norm in this proof. We first treat the $\theta \cdot \nabla$ term. By Eqs. (4.2)-(4.3) we have

$$
\begin{aligned}
& H_{0}^{-\frac{1}{2}}(-21 \theta \cdot \nabla) H_{0}^{\frac{1}{2}}\left(\lambda-H_{0}\right)^{-1}-P_{N} H_{0}^{-\frac{1}{2}}(-21 \theta \cdot \nabla) H_{0}^{\frac{1}{2}}\left(\lambda-H_{0}\right)^{-1} P_{N} \\
& \quad=\operatorname{diag}\left(\frac{2 \theta \cdot k_{j}}{\lambda-1-\left|k_{j}\right|^{2}} ; j>N\right)
\end{aligned}
$$

and by (4.4) we can estimate the above as

$$
\begin{aligned}
\left\|\operatorname{diag}\left(\frac{2 \theta \cdot k_{j}}{\lambda-1-\left|k_{j}\right|^{2}} ; j>N\right)\right\|_{\mathcal{C}_{s}} & \leq \frac{2}{\pi}|\theta| C_{\lambda}\left(\sum_{j=N+1}^{\infty} j^{-\frac{s}{d}}\right)^{\frac{1}{s}} \\
& \leq \frac{2}{\pi}|\theta| C_{\lambda}\left(\int_{N}^{\infty} t^{-\frac{s}{d}} d t\right)^{\frac{1}{s}} \\
& \leq \frac{2}{\pi}|\theta| C_{\lambda}\left(\frac{N^{-\frac{s}{d}+1}}{\frac{s}{d}-1}\right)^{\frac{1}{s}} \\
& =\frac{2|\theta| C_{\lambda}}{\pi\left(\frac{s}{d}-1\right)^{\frac{1}{s}}} N^{-\frac{1}{d}+\frac{1}{s}}
\end{aligned}
$$

To estimate the next term, we denote $W_{n}:=|\theta|^{2}-1+V^{\text {appr, } n}$ and $W:=|\theta|^{2}-1+V$ for brevity, and note that the diagonal operators $H_{0}^{-\frac{1}{2}}$ and $\left(\lambda-H_{0}\right)^{-1}$ commute with $P_{N}$. Thus we have $P_{N} H_{0}^{-\frac{1}{2}} W_{n} H_{0}^{\frac{1}{2}}\left(\lambda-H_{0}\right)^{-1} P_{N}=H_{0}^{-\frac{1}{2}} P_{N} W_{n} P_{N} H_{0}^{\frac{1}{2}}\left(\lambda-H_{0}\right)^{-1}$. 
Then we calculate

$$
\begin{aligned}
\left\|H_{0}^{-\frac{1}{2}}\left(W-P_{N} W_{n} P_{N}\right) H_{0}^{\frac{1}{2}}\left(\lambda-H_{0}\right)^{-1}\right\|_{\mathcal{C}_{s}} \\
\leq\left\|H_{0}^{-\frac{1}{2}}\left(W-P_{N} W P_{N}\right) H_{0}^{\frac{1}{2}}\left(\lambda-H_{0}\right)^{-1}\right\|_{\mathcal{C}_{s}} \\
\quad+\left\|H_{0}^{-\frac{1}{2}} P_{N}\left(W-W_{n}\right) P_{N} H_{0}^{\frac{1}{2}}\left(\lambda-H_{0}\right)^{-1}\right\|_{\mathcal{C}_{s}} \\
\leq\left\|H_{0}^{-\frac{1}{2}}\left(W-P_{N} W P_{N}\right) H_{0}^{\frac{1}{2}}\left(\lambda-H_{0}\right)^{-1}\right\|_{\mathcal{C}_{s}} \\
\quad+\left\|H_{0}^{-\frac{1}{2}} P_{N}\left(V-V^{\mathrm{appr}, n}\right) P_{N} H_{0}^{\frac{1}{2}}\left(\lambda-H_{0}\right)^{-1}\right\|_{\mathcal{C}_{s}} .
\end{aligned}
$$

Let us first consider the second term on the right-hand side of (4.11).

$$
\begin{aligned}
& \left\|H_{0}^{-\frac{1}{2}} P_{N}\left(V-V^{\mathrm{appr}, n}\right) P_{N} H_{0}^{\frac{1}{2}}\left(\lambda-H_{0}\right)^{-1}\right\|_{\mathcal{C}_{s}} \\
& \leq\left\|H_{0}^{-\frac{1}{2}}\right\|\left\|P_{N}\left(V-V^{\mathrm{appr}, n}\right) P_{N}\right\|\left\|H_{0}^{\frac{1}{2}}\left(\lambda-H_{0}\right)^{-1}\right\|_{\mathcal{C}_{s}} \\
& \quad \leq \frac{2}{\pi} C_{\lambda}\left(1-\frac{d}{s}\right)^{-\frac{1}{s}}\left\|P_{N}\left(V-V^{\mathrm{appr}, n}\right) P_{N}\right\| \\
& \quad \leq \frac{2}{\pi} C_{\lambda}\left(1-\frac{d}{s}\right)^{-\frac{1}{s}} N \frac{2 n^{\frac{d}{p}-1}}{1-\frac{d}{p}}\left(1+\sup _{j \leq N}\left|k_{j}\right|\right)\|V\|_{W^{1, p}\left((0,1)^{d}\right)} \\
& \quad \leq \frac{2}{\pi} C_{\lambda}\left(1-\frac{d}{s}\right)^{-\frac{1}{s}} N \frac{2 n^{\frac{d}{p}-1}}{1-\frac{d}{p}}\left(1+\pi d^{\frac{1}{2}} N^{\frac{1}{d}}\right)\|V\|_{W^{1, p}\left((0,1)^{d}\right)}
\end{aligned}
$$

where the fourth line follows from (4.9), with a similar calculation as in (4.6). To simplify notation, we collect all constants independent of $\lambda, n, N$ into one and write

$$
\left\|H_{0}^{-\frac{1}{2}} P_{N}\left(V-V^{\mathrm{appr}, n}\right) P_{N} H_{0}^{\frac{1}{2}}\left(\lambda-H_{0}\right)^{-1}\right\|_{\mathcal{C}_{s}} \leq C_{\lambda} C_{s, p, d}^{2} \frac{N^{1+1 / d}}{n^{1-d / p}}\|V\|_{W^{1, p}\left((0,1)^{d}\right)}
$$

Next we turn to the first term on the right-hand side of (4.11). We add and subtract $P_{N} W$ and use the triangle inequality to obtain

$$
\begin{aligned}
& \left\|H_{0}^{-\frac{1}{2}}\left(W-P_{N} W P_{N}\right) H_{0}^{\frac{1}{2}}\left(\lambda-H_{0}\right)^{-1}\right\|_{\mathcal{C}_{s}} \\
& \leq\left\|H_{0}^{-\frac{1}{2}}\left(W-P_{N} W\right) H_{0}^{\frac{1}{2}}\left(\lambda-H_{0}\right)^{-1}\right\|_{\mathcal{C}_{s}} \\
& \quad+\left\|H_{0}^{-\frac{1}{2}}\left(P_{N} W-P_{N} W P_{N}\right) H_{0}^{\frac{1}{2}}\left(\lambda-H_{0}\right)^{-1}\right\|_{\mathcal{C}_{s}}
\end{aligned}
$$




$$
\begin{aligned}
= & \left\|H_{0}^{-\frac{1}{2}}\left(I-P_{N}\right) W H_{0}^{\frac{1}{2}}\left(\lambda-H_{0}\right)^{-1}\right\|_{\mathcal{C}_{s}} \\
& +\left\|H_{0}^{-\frac{1}{2}} P_{N} W\left(I-P_{N}\right) H_{0}^{\frac{1}{2}}\left(\lambda-H_{0}\right)^{-1}\right\|_{\mathcal{C}_{s}} \\
\leq & \left\|H_{0}^{-\frac{1}{2}}\left(I-P_{N}\right)\right\|_{\mathcal{C}_{s}}\left\|H_{0}^{\frac{1}{2}}\left(\lambda-H_{0}\right)^{-1}\right\|\|W\| \\
& +\left\|H_{0}^{-\frac{1}{2}}\right\|\left\|\left(I-P_{N}\right) H_{0}^{\frac{1}{2}}\left(\lambda-H_{0}\right)^{-1}\right\|_{\mathcal{C}_{s}}\|W\| .
\end{aligned}
$$

Next we note that, by (4.2)-(4.3), $\left\|H_{0}^{-\frac{1}{2}}\right\|=1$ and $\left\|H_{0}^{\frac{1}{2}}\left(\lambda-H_{0}\right)^{-1}\right\| \leq 2 C_{\lambda}$. Therefore

$$
\begin{aligned}
& \left\|H_{0}^{-\frac{1}{2}}\left(W-P_{N} W P_{N}\right) H_{0}^{\frac{1}{2}}\left(\lambda-H_{0}\right)^{-1}\right\|_{\mathcal{C}_{s}} \\
& \quad \leq\left(2 C_{\lambda}\left\|H_{0}^{-\frac{1}{2}}\left(I-P_{N}\right)\right\|_{\mathcal{C}_{s}}+\left\|\left(I-P_{N}\right) H_{0}^{\frac{1}{2}}\left(\lambda-H_{0}\right)^{-1}\right\|_{\mathcal{C}_{s}}\right)\|W\| .
\end{aligned}
$$

Finally, we employ (4.2)-(4.3) again to estimate the finite section error in (4.13). A straightforward calculation shows that

$$
\begin{aligned}
\left\|H_{0}^{-\frac{1}{2}}\left(I-P_{N}\right)\right\|_{\mathcal{C}_{s}} & \leq \pi^{-1} \frac{N^{\frac{1}{s}-\frac{1}{d}}}{\left(\frac{s}{d}-1\right)^{1 / s}}, \\
\left\|\left(I-P_{N}\right) H_{0}^{\frac{1}{2}}\left(\lambda-H_{0}\right)^{-1}\right\|_{\mathcal{C}_{s}} & \leq \frac{2}{\pi} C_{\lambda} \frac{N^{\frac{1}{s}-\frac{1}{d}}}{\left(\frac{s}{d}-1\right)^{1 / s}} .
\end{aligned}
$$

Using these bounds in (4.13), we finally obtain the error estimate

$$
\begin{aligned}
& \left\|H_{0}^{-\frac{1}{2}}\left(W-P_{N} W P_{N}\right) H_{0}^{\frac{1}{2}}\left(\lambda-H_{0}\right)^{-1}\right\|_{\mathcal{C}_{s}} \\
& \quad \leq \frac{4 C_{\lambda} N^{\frac{1}{s}-\frac{1}{d}}}{\pi\left(\frac{s}{d}-1\right)^{1 / s}}\|W\| \\
& \leq \frac{4 C_{\lambda} N^{\frac{1}{s}-\frac{1}{d}}}{\pi\left(\frac{s}{d}-1\right)^{1 / s}}\left(\left.|| \theta\right|^{2}-1 \mid+\|V\|_{L^{\infty}\left((0,1)^{d}\right)}\right) .
\end{aligned}
$$

Combining (4.10), (4.12) and (4.14) yields the assertion with constants

$$
\begin{aligned}
C_{s, d}^{1} & =\frac{2}{\pi\left(\frac{s}{d}-1\right)^{\frac{1}{s}}}, \\
C_{s, p, d}^{2} & =\frac{2}{\pi}\left(1-\frac{d}{s}\right)^{-\frac{1}{s}} \frac{2}{1-\frac{d}{p}}\left(1+\pi d^{1 / 2}\right), \\
C_{s, d}^{3} & =\frac{4}{\pi\left(\frac{s}{d}-1\right)^{1 / s}} .
\end{aligned}
$$




\subsection{Proof of Theorem 4.1}

In this section we construct an algorithm $\Gamma_{N}(V)$, which approximates $\sigma(-\Delta+V)$ as $N \rightarrow \infty$. The output of $\Gamma_{N}$ consists of two parts $\Gamma_{n}^{z}$ and $\tilde{\Gamma}_{n}^{z}$, which we call algorithmic components. We first recall a result on Lipschitz continuity of perturbation determinants.

Theorem 4.7 ( [35, Thm. 6.5]) For $m \in \mathbb{N}$, denote by $\operatorname{det}_{m}$ the perturbation determinant on $\mathcal{C}_{m}$ (cf. [14, Sec. XI.9]). Then there exists a constant $c_{m}$ such that

$$
\left|\operatorname{det}_{m}(I-A)-\operatorname{det}_{m}(I-B)\right| \leq e^{c_{m}\left(1+\|A\|_{\mathcal{C}_{m}}+\|B\|_{\mathcal{C}_{m}}\right)}\|A-B\|_{\mathcal{C}_{m}}
$$

for all $A, B \in \mathcal{C}_{m}$. Moreover, one has $c_{m} \leq e(2+\log (m))$.

Next, we define

Definition 4.8 (First algorithmic component) Let $p>d, V \in \Omega_{p}^{\mathrm{Sch}}$ and $z_{0} \in \mathbb{C}$. For $N \in \mathbb{N}$ let $\Theta_{N}=\left(\theta_{1}^{(N)}, \ldots, \theta_{N}^{(N)}\right)$ be a linearly spaced lattice in $[0,2 \pi]^{d}$ and let $L_{N}:=\frac{1}{N}(\mathbb{Z}+1 \mathbb{Z}) \cap Q_{z_{0}}$, where $Q_{z_{0}}:=\left\{z \in \mathbb{C}|| \operatorname{Im}\left(z-z_{0}\right)|,| \operatorname{Re}\left(z-z_{0}\right) \mid \leq \frac{1}{2}\right\}$. Then we let $n(N):=N^{\lceil\alpha\rceil}$, with $\alpha=\frac{1+2 d^{-1}-p^{-1}}{1-p^{-1} d},{ }^{2}$ and define

$$
\Gamma_{N}^{z_{0}}(V):=\bigcup_{i=1}^{N}\left\{z \in L_{N}|| \operatorname{det}_{\lceil p\rceil}\left(I-P_{N} K_{n(N)}^{\mathrm{appr}}\left(z, \theta_{i}^{(N)}\right) P_{N}\right) \mid \leq N^{-\left(\frac{1}{2 d}-\frac{1}{2 p}\right)}\right\} .
$$

Note that for every $N \in \mathbb{N}, \Gamma_{N}^{z_{0}}(V)$ can be computed from the information in $\Lambda$ using finitely many algebraic operations (recall in particular the approximated Fourier coefficients (4.8)). Therefore, every $\Gamma_{N}^{z_{0}}$ defines an arithmetic algorithm in the sense of Definition 2.3.

Proposition 4.9 (Convergence of first algorithmic component) Let $z_{0} \in \mathbb{C}$ and let $V \in \Omega_{p}^{\mathrm{Sch}}$. The following statements hold.

(i) For any sequence $z_{N} \in \Gamma_{N}^{z_{0}}(V)$ with $z_{N} \rightarrow z \in Q_{z_{0}} \backslash \bigcup_{j}\left(1+\left|k_{j}\right|^{2}\right)$ one has $z \in \sigma(-\Delta+V)$.

(ii) For any $z \in\left(\sigma(-\Delta+V) \cap Q_{z_{0}}\right) \backslash \bigcup_{j}\left(1+\left|k_{j}\right|^{2}\right)$ there exists a sequence $z_{N} \in$ $\Gamma_{N}^{z_{0}}(V)$ with $z_{N} \rightarrow z$ as $N \rightarrow+\infty$.

(iii) Let $V \in \Omega_{p, M}^{\mathrm{Sch}}$. For any given $\varepsilon, \delta>0$ one has $\left(\sigma(-\Delta+V) \cap Q_{z_{0}}\right) \backslash$ $B_{\delta}\left(\bigcup_{j}\left(1+\left|k_{j}\right|^{2}\right)\right) \subset B_{\varepsilon}\left(\Gamma_{N}^{z_{0}}(V)\right)$ as soon as $N>\max \left\{\varepsilon^{-1}, N_{\delta, z_{0}}\right\}$, where

$$
N_{\delta, z_{0}}=\left[C_{\delta, z_{0}}^{\mathrm{Lip}}+G\left(\left|z_{0}\right|+1\right) \delta^{-1}\right]^{\frac{2}{1 / p+1 / d}}
$$

and $C_{\delta, z_{0}}^{\mathrm{Lip}}, G$ are explicit constants, which can be computed in finitely many operations from $z_{0}, \delta, s, p, d$ and the a priori bound $M$ for $\|V\|_{W^{1, p}}$.

$\overline{2}$ The exponent $\alpha$ is chosen such that $N^{1+1 / d} / n^{1-d / p} \leq N^{1 / p-1 / d}$ (cf. Lemma (4.6)). 
We defer the proof of Proposition 4.9 to Appendix C. The algorithmic component $\Gamma_{N}^{z_{0}}$ defined in (4.15) on its own is not sufficient to prove Theorem 4.1, because it does not approximate any eigenvalues lying in the set $\bigcup_{j}\left(1+\left|k_{j}\right|^{2}\right)$. This is ultimately due to the decomposition (4.1) into $H_{0}$ and $B(\theta)$. In order to solve this issue, we define a second algorithmic component based on a different decomposition. We define

$$
\begin{aligned}
\tilde{H}_{0} & :=-\Delta+2, & \operatorname{dom}\left(\tilde{H}_{0}\right) & =H_{\text {per }}^{2}\left((0,1)^{d}\right) \\
\tilde{B}(\theta) & :=-21 \theta \cdot \nabla+|\theta|^{2}-2+V, & \operatorname{dom}(\tilde{B}(\theta)) & =H_{\text {per }}^{1}\left((0,1)^{d}\right) .
\end{aligned}
$$

Then, obviously, $\tilde{H}_{0}+\tilde{B}(\theta)=H(\theta)$ and by the same calculation as in Sect. 4.1.1,

$$
\lambda \in \mathbb{C} \backslash \sigma\left(\tilde{H}_{0}\right) \text { is in } \sigma(H(\theta)) \Leftrightarrow 1 \in \sigma\left(\tilde{H}_{0}^{-\frac{1}{2}} \tilde{B}(\theta) \tilde{H}_{0}^{\frac{1}{2}}\left(\lambda-\tilde{H}_{0}\right)^{-1}\right) .
$$

Moreover, we have $\sigma\left(\tilde{H}_{0}\right)=\bigcup_{j}\left(2+\left|k_{j}\right|^{2}\right)$ and

Lemma 4.10 One has

$$
\sigma\left(H_{0}\right) \cap \sigma\left(\tilde{H}_{0}\right)=\emptyset .
$$

Proof The assertion is equivalent to the equation $2+(2 \pi)^{2} \sum_{i=1}^{d} \tilde{n}_{i}^{2}=1+$ $(2 \pi)^{2} \sum_{i=1}^{d} n_{i}^{2}$ having no solutions for any integers $n_{i}, \tilde{n}_{i}$, which becomes $1=$ $(2 \pi)^{2} \sum_{i=1}^{d}\left(n_{i}^{2}-\tilde{n}_{i}^{2}\right)$. Clearly the right-hand side of the last equation is always irrational or zero, while the left hand side is always nonzero rational. Thus no solution exists.

Subsection 4.1 and Proposition 4.9 carry over trivially to the decomposition $\tilde{H}_{0}, \tilde{B}(\theta)$. Analogously to (4.15) we define the new algorithm

Definition 4.11 (Second algorithmic component) Let $p>d, V \in \Omega_{p}^{\mathrm{Sch}}$ and $z_{0} \in \mathbb{C}$. For $N \in \mathbb{N}$ let $\Theta_{N}=\left(\theta_{1}^{(N)}, \ldots, \theta_{N}^{(N)}\right)$ be a linearly spaced lattice in $[0,2 \pi]^{d}$ and let $L_{N}:=\frac{1}{N}(\mathbb{Z}+1 \mathbb{Z}) \cap Q_{z_{0}}$, where $Q_{z_{0}}:=\left\{z \in \mathbb{C}|| \operatorname{Im}\left(z-z_{0}\right)|,| \operatorname{Re}\left(z-z_{0}\right) \mid \leq \frac{1}{2}\right\}$. Then we choose $n(N):=N^{\lceil\alpha\rceil}$ (with $\alpha$ as in Definition 4.8) and define

$$
\tilde{\Gamma}_{N}^{z_{0}}(V):=\bigcup_{i=1}^{N}\left\{z \in L_{N}|| \operatorname{det}_{\lceil p\urcorner}\left(I-P_{N} \tilde{K}_{n(N)}^{\mathrm{appr}}\left(z, \theta_{i}^{(N)}\right) P_{N}\right) \mid \leq N^{-\left(\frac{1}{2 d}-\frac{1}{2 p}\right)}\right\}
$$

where now

$$
\tilde{K}_{n}^{\text {appr }}(\lambda, \theta):=\tilde{H}_{0}^{-\frac{1}{2}}\left(-21 \theta \cdot \nabla+|\theta|^{2}-2+V^{\text {appr }, n}\right) \tilde{H}_{0}^{\frac{1}{2}}\left(\lambda-\tilde{H}_{0}\right)^{-1} .
$$

From the proofs in Subsection 4.1 and Proposition 4.9 we immediately obtain the following convergence result. 
Proposition 4.12 (Convergence of second algorithmic component) Let $z_{0} \in \mathbb{C}$ and let $V \in \Omega_{p}^{\text {Sch }}$. The following statements hold.

(i) For any sequence $z_{N} \in \tilde{\Gamma}_{N}^{z_{0}}(V)$ with $z_{N} \rightarrow z \in Q_{z_{0}} \backslash \bigcup_{j}\left(2+\left|k_{j}\right|^{2}\right)$ one has $z \in \sigma(-\Delta+V)$.

(ii) For any $z \in\left(\sigma(-\Delta+V) \cap Q_{z_{0}}\right) \backslash \bigcup_{j}\left(2+\left|k_{j}\right|^{2}\right)$ there exists a sequence $z_{N} \in$ $\tilde{\Gamma}_{N}^{z_{0}}(V)$ with $z_{N} \rightarrow z$ as $N \rightarrow+\infty$.

(iii) Let $V \in \Omega_{p, M}^{\mathrm{Sch}}$. For any given $\varepsilon, \delta>0$ one has $\left(\sigma(-\Delta+V) \cap Q_{z_{0}}\right) \backslash$ $B_{\delta}\left(\bigcup_{j}\left(2+\left|k_{j}\right|^{2}\right)\right) \subset B_{\varepsilon}\left(\tilde{\Gamma}_{N}^{z_{0}}(V)\right)$ as soon as $N>\max \left\{\varepsilon^{-1}, N_{\delta, z_{0}}\right\}$, where $N_{\delta, z_{0}}$ was defined in (4.16).

Armed with the algorithmic components (4.15) and (4.18), we are finally able to define the main algorithm:

Definition 4.13 (Main Algorithm) Let $p>d, V \in \Omega_{p}^{\mathrm{Sch}}$, and choose a numbering $\left\{Z_{j}\right\}_{j \in \mathbb{N}}$ of $\mathbb{Z}+1 \mathbb{Z}$ such that $\left|Z_{i}\right| \leq\left|Z_{j}\right|$ for $i \leq j$. For $N \in \mathbb{N}$ define

$$
\Gamma_{N}(V):=\bigcup_{i=1}^{N}\left(\Gamma_{N}^{Z_{i}}(V) \cup \tilde{\Gamma}_{N}^{Z_{i}}(V)\right) .
$$

Combining Propositions 4.9 and 4.12, we can complete the proof of Theorem 4.1:

Proof (Proof of Theorem 4.1) Let $p>d$. For any $V \in \Omega_{p}^{\text {Sch }}$ we need to show that

$$
d_{\mathrm{AW}}\left(\Gamma_{N}(V), \sigma(-\Delta+V)\right) \rightarrow 0 .
$$

Indeed, by Propositions 4.9 and 4.12, the following holds

(a) For any sequence $z_{N} \in \Gamma_{N}(V)$ with $z_{N} \rightarrow z \in \mathbb{C}$ one has $z \in \sigma(-\Delta+V)$.

(b) For any $z \in \sigma(-\Delta+V)$ there exists a sequence $z_{N} \in \Gamma_{N}(V)$ with $z_{N} \rightarrow z$ as $N \rightarrow+\infty$.

Attouch-Wets convergence now follows from a standard argument, cf. [32, Prop. 2.8].

\subsection{Proof of Theorem 1.2}

We can finally prove Theorem 1.2.

Proof Proof of part (i) The a priori knowledge of $p$ can be removed by slightly modifying the algorithmic components (4.15) and (4.18). An algorithm that achieves $\mathrm{SCI}=1$ is obtained by modifying the determinant threshold in (4.15) and (4.18). If the cutoff $N^{-\left(\frac{1}{2 d}-\frac{1}{2 p}\right)}$ is replaced by $\log (N)^{-1}$ and the discretization width $n(N)=N^{\lceil\alpha\rceil}$ is replaced by $n(N):=e^{N}$, the proof of Propositions 4.9 and 4.12 is valid independently of the value of $p$ (cf. Eq. (C.3)).

Proof of part (ii). Fix $R>0$. According to our choice of numbering $\left\{Z_{i}\right\}_{i \in \mathbb{N}}$ we have $\left|Z_{i}\right|>R$ for all $i>4 R^{2}$ and thus $B_{R}(0) \subset \bigcup_{i \leq 4 R^{2}} Q_{Z_{i}}$. Let $\delta_{R}=$ 
$\frac{1}{2} \min \left\{|x-y|\left|x \in \sigma\left(H_{0}\right), y \in \sigma\left(\tilde{H}_{0}\right),\right| x|| y \mid, \leq R\right\}$, which is greater than 0 by Lemma 4.10. This choice implies that removing $\delta_{R}$-neighborhoods of the free spectra does not actually restrict our domain of computation:

$$
\left(B_{R}(0) \backslash B_{\delta_{R}}\left(\sigma\left(H_{0}\right)\right)\right) \cup\left(B_{R}(0) \backslash B_{\delta_{R}}\left(\sigma\left(\tilde{H}_{0}\right)\right)\right)=B_{R}(0)
$$

for all $R>0$. Now let $\varepsilon>0$. By Propositions 4.9(iii) and 4.12(iii) we have

$$
\sigma(-\Delta+V) \cap B_{R}(0) \subset B_{\varepsilon}\left(\Gamma_{N}(V)\right)
$$

as soon as $N>\max \left\{4 R^{2}, \varepsilon^{-1}, N_{R}\right\}$, where $N_{R}=\max \left\{N_{\delta_{R}, Z_{i}} \mid i \leq 4 R^{2}\right\}$ (recall $N_{\delta, z}$ from (4.16)). We recall that

- the lower bound $4 R^{2}$ ensures that $B_{R}(0)$ is covered by the search regions $Q_{Z_{i}}$;

- the lower bound $\varepsilon^{-1}$ ensures that for every $z \in \sigma(-\Delta+V)$ there exists $z_{N} \in L_{N}$ with $\left|z-z_{N}\right|<\varepsilon$;

- the lower bound $N_{R}$ ensures that $z_{N} \in \Gamma_{N}(V)$.

To conclude the proof of part (ii) (i.e., that $\Omega_{p, M}^{\mathrm{Sch}} \in \Pi_{1}$ ), we need a set $X_{k}$ as in Definition 2.7. To this end, for $V \in \Omega_{p, M}^{\mathrm{Sch}}$ and $k \in \mathbb{N}$, choose $N(k):=\max \left\{4 k^{2}, 2^{k}, N_{k}\right\}$ and define

$$
X_{k}:=B_{2^{-k}}\left(\Gamma_{N(k)}(V)\right) \cup\left(\mathbb{C} \backslash B_{k}(0)\right)
$$

Then by the definition of the Attouch-Wets distance $d_{\text {AW }}$ (cf. Definition A.2) one has

$$
\begin{aligned}
d_{\mathrm{AW}}\left(\Gamma_{N(k)}(V), X_{k}\right)= & \sum_{n=1}^{\infty} 2^{-n} \min \left\{1, \sup _{\substack{p \in \mathbb{C} \\
|p|<n}}\left|\inf _{a \in \Gamma_{N(k)}(V)}\right| a-p\left|-\inf _{b \in X_{k}}\right| b-p||\right\} \\
\leq & \sum_{n=1}^{k} 2^{-n} \min \left\{1, \sup _{\substack{p \in \mathbb{C} \\
|p|<k}}\left|\inf _{a \in \Gamma_{N(k)}(V)}\right| a-p\left|-\inf _{b \in X_{k}}\right| b-p||\right\} \\
& +\sum_{n=k+1}^{\infty} 2^{-n} \\
\leq & 2^{-k} \sum_{n=1}^{k} 2^{-n}+2^{-k} \\
\leq & 2^{-k+1}
\end{aligned}
$$

where the third line follows from the definition of $X_{k}$. Moreover, by (4.19) (with $\varepsilon=2^{-k}$ and $R=k$ ) we have

$$
\sigma(-\Delta+V) \subset X_{k}
$$

for all $k \in \mathbb{N}$. These facts, together with step (i) imply $\Omega_{p, M}^{\mathrm{Sch}} \in \Pi_{1}$. 


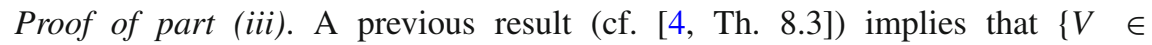
$\Omega_{p, M}^{\mathrm{Sch}} \mid V$ real-valued $\} \in \Sigma_{1}$. Combined with Theorem 1.2(ii) and [4, Prop. 6.15] we obtain

$$
\left\{V \in \Omega_{p, M}^{\mathrm{Sch}} \mid V \text { real-valued }\right\} \in \Delta_{1}
$$

i.e. if the potential is periodic and real-valued, the spectrum can be computed with full error control.

Remark 4.14 In view of Theorems 3.3 and 4.1 it is natural to ask whether one might have $\Omega_{p, M}^{\mathrm{Sch}} \in \Sigma_{1}$ (and therefore $\Omega_{p, M}^{\mathrm{Sch}} \in \Delta_{1}$ ). This is indeed a nontrivial open problem. Recalling the proof of Theorem 3.3 (in particular (3.11)), the error bound for the approximation "from below" used the fact that $\operatorname{det}(z I-A(\theta))$ is a polynomial. In the proof of Theorem 4.1 on the other hand, the function $\operatorname{det}_{\lceil p\rceil}(I-K(z, \theta))$, whose zeros must be approximated, is only known to be analytic. Obtaining $\Sigma_{1}$ classification amounts to obtaining explicit upper bounds on the width of the zeros of this analytic function. These cannot be deduced in any straightforward way from the values of the potential $V$.

\section{Sometimes two limits are necessary}

In Sect. 2 we described a complicated construction called towers of algorithms, where more than one successive limit is required to correctly perform certain computations. In this section we exhibit this phenomenon first hand: we prove Theorem 1.3 which is rephrased in the language of SCI as Theorem 5.1 below. This theorem shows that there exists a class of potentials (which are less smooth, yet can be evaluated at any point) for which there do not exist algorithms that can approximate the associated spectral problem in a single limit. That is, SCI $>1$. We are able, though, to construct an arithmetic algorithm which converges by taking two successive limits. That is, $\mathrm{SCI}=2$. This class contains potentials which are allowed to have a singularity at a single point $x_{0} \in(0,1)$, but are otherwise smooth:

$$
\begin{gathered}
\Omega_{x_{0}}^{\mathrm{Sch}}:=\left\{V: \mathbb{R} \rightarrow \mathbb{R} \mid V \text { is 1-periodic, } V\left(x_{0}\right)=0\right. \text { and } \\
\left.\left.V\right|_{[0,1]} \in L^{2}([0,1]) \cap C^{\infty}\left([0,1] \backslash\left\{x_{0}\right\}\right)\right\} .
\end{gathered}
$$

We emphasize that $\Omega_{x_{0}}^{\text {Sch }}$ contains only real-valued functions and that the pointwise evaluation $V \mapsto V(x)$ is well-defined for all $x \in \mathbb{R}$. Moreover, we remark that by [29, Th. XIII.96] the Schrödinger operator $-\Delta+V$ is well-defined and selfadjoint with domain $H^{2}(\mathbb{R})$. We shall therefore consider the computational problem 


$$
\left\{\begin{aligned}
\Omega & =\Omega_{x_{0}}^{\text {Sch }} \\
\mathcal{M} & =\left(\{K \subset \mathbb{C} \mid K \text { closed }\}, d_{\mathrm{AW}}\right) \\
\Lambda & =\left\{V \mapsto V(x) \mid x \in \mathbb{R}^{d}\right\} \\
\Xi: & \Omega \rightarrow \mathcal{M} ; \quad V \mapsto \sigma\left(-\partial_{x}^{2}+V\right),
\end{aligned}\right.
$$

and shall prove

Theorem 5.1 The computational problem (5.1) has SCI = 2 (equivalently, it belongs to $\left.\Delta_{3} \backslash \Delta_{2}\right)$.

The proof of Theorem 5.1 has two parts. To prove SCI $\leq 2$ we construct an explicit tower of algorithms that computes the spectrum in two limits (cf. Definition 2.4). The proof of SCI $>1$ is by contradiction. We assume the existence of a sequence $\left\{\Gamma_{n}\right\}_{n \in \mathbb{N}}$ with $\Gamma_{n}(V) \rightarrow \sigma\left(-\partial_{x}^{2}+V\right)$ for every $V \in \Omega_{x_{0}}^{\text {Sch }}$ and via a diagonal process construct a potential $V \in \Omega_{x_{0}}^{\text {Sch }}$ such that $\Gamma_{n}(V) \nrightarrow \sigma\left(-\partial_{x}^{2}+V\right)$, yielding the desired contradiction.

\subsection{Lemmas}

We first collect some technical lemmas that will be necessary for the proof.

Lemma 5.2 Denote by $W(H)$ the numerical range of an operator $H$. Let $V \in \Omega_{x_{0}}^{\text {Sch }}$. There exists $\delta>0$ such that inf $W\left(-\partial_{x}^{2}+V\right) \geq-\frac{1}{4}$ whenever $\|V\|_{L^{1}([0,1])}<\delta$.

Proof Choose a partition of unity $\left\{\chi_{i}\right\}_{i \in \mathbb{Z}} \operatorname{such}$ that $\operatorname{supp}\left(\chi_{i}\right) \subset[i-1, i+1]$ and $\sup _{i \in \mathbb{Z}}\left\|\chi_{i}\right\|_{W^{1, \infty}}<+\infty$. Then for any $\phi \in C_{0}^{\infty}(\mathbb{R})$ we have

$$
\begin{aligned}
\int_{\mathbb{R}} V(x)|\phi(x)|^{2} d x & =\sum_{i \in \mathbb{Z}} \int_{\mathbb{R}} V(x) \chi_{i}(x)|\phi(x)|^{2} d x \\
& =\sum_{i \in \mathbb{Z}} \int_{i-1}^{i+1} V(x) \chi_{i}(x)|\phi(x)|^{2} d x \\
& =\sum_{i \in \mathbb{Z}} \int_{-1}^{1} V(x) \chi_{i}(x-i)|\phi(x-i)|^{2} d x,
\end{aligned}
$$

where the last line follows from periodicity of $V$. Moreover, we have

$$
\begin{aligned}
\left.\left|\int_{-1}^{1} V(x) \chi_{i}(x-i)\right| \phi(x-i)\right|^{2} d x \mid & \leq\|V\|_{L^{1}([-1,1])}\left\|\chi_{i}(\cdot-i)|\phi(\cdot-i)|^{2}\right\|_{L^{\infty}([-1,1])} \\
& \leq C\|V\|_{L^{1}([0,1])}\left\|\chi_{i}(\cdot-i)|\phi(\cdot-i)|^{2}\right\|_{W^{1,1}([-1,1])} \\
& \leq C\|V\|_{L^{1}([0,1])}\left\|\chi_{i}\right\|_{W^{1, \infty}}\|\phi(\cdot-i)\|_{H^{1}([-1,1])}^{2},
\end{aligned}
$$


where the second line follows from the Sobolev embedding $W^{1,1} \hookrightarrow L^{\infty}$ (cf. [9, Th. 8.8]) and the third follows by the product rule and Hölder's inequality. Summing (5.2) over $i$ we obtain

$$
\begin{aligned}
\left.\left|\int_{\mathbb{R}} V(x)\right| \phi(x)\right|^{2} d x \mid & \leq C\|V\|_{L^{1}([0,1])} \sum_{i \in \mathbb{Z}}\left\|\chi_{i}\right\|_{W^{1, \infty}}\|\phi(\cdot-i)\|_{H^{1}([-1,1])}^{2} \\
& \leq 2 C\|V\|_{L^{1}([0,1])}\left(\sup _{i \in \mathbb{Z}}\left\|\chi_{i}\right\|_{W^{1, \infty}}\right)\|\phi\|_{H^{1}(\mathbb{R})}^{2} \\
& \leq C^{\prime}\|V\|_{L^{1}([0,1])}\|\phi\|_{H^{1}(\mathbb{R})}^{2},
\end{aligned}
$$

where $C^{\prime}=2 C \sup _{i \in \mathbb{Z}}\left\|\chi_{i}\right\|_{W^{1, \infty}}$ is finite by choice of the functions $\chi_{i}$. Turning to the numerical range, we have for $\phi \in C_{0}^{\infty}(\mathbb{R})$

$$
\begin{aligned}
\left\langle\left(-\partial_{x}^{2}+V\right) \phi, \phi\right\rangle_{L^{2}(\mathbb{R})} & =\int_{\mathbb{R}}\left(\left|\phi^{\prime}\right|^{2}+V|\phi|^{2}\right) d x \\
& \geq\left\|\phi^{\prime}\right\|_{L^{2}(\mathbb{R})}^{2}-C^{\prime}\|V\|_{L^{1}([0,1])}\|\phi\|_{H^{1}(\mathbb{R})}^{2} \\
& =\left(1-C^{\prime}\|V\|_{L^{1}([0,1])}\right)\left\|\phi^{\prime}\right\|_{L^{2}(\mathbb{R})}^{2}-C^{\prime}\|V\|_{L^{1}([0,1])}\|\phi\|_{L^{2}(\mathbb{R})}^{2}
\end{aligned}
$$

where the second line follows from (5.3). Finally, let $\delta:=\left(4 C^{\prime}\right)^{-1}$. Then if $\|V\|_{L^{1}([0,1])}<\delta$ one has

$$
\left\langle\left(-\partial_{x}^{2}+V\right) \phi, \phi\right\rangle_{L^{2}(\mathbb{R})} \geq \frac{3}{4}\left\|\phi^{\prime}\right\|_{L^{2}(\mathbb{R})}^{2}-\frac{1}{4}\|\phi\|_{L^{2}(\mathbb{R})}^{2} \geq-\frac{1}{4}\|\phi\|_{L^{2}(\mathbb{R})}^{2}
$$

and the proof is complete.

Lemma 5.3 For $h>0, q_{1}, q_{2} \in[0,1]$ with $0<q_{1}<q_{2}<1$, define the periodic step function

$$
V_{h, q_{1}, q_{2}}(x)= \begin{cases}-h & x \in\left[q_{1}, q_{2}\right] \\ 0 & x \in\left([0,1] \backslash\left[q_{1}, q_{2}\right]\right)\end{cases}
$$

Then for every $\tilde{V} \in \Omega_{x_{0}}^{\text {Sch }}$ with $\tilde{V} \leq 0$ one has

$$
\inf W\left(-\partial_{x}^{2}+\tilde{V}+V_{h, q_{1}, q_{2}}\right) \leq-\left(q_{2}-q_{1}\right) h
$$

Proof Define the sequence of test functions

$$
\phi_{n}(x)= \begin{cases}\frac{1}{\sqrt{2 n}} & x \in[-n, n] \\ \text { linear to } 0 & x \in[-n-1,-n) \cup(n, n+1] \\ 0 & \text { otherwise }\end{cases}
$$


We note that $\left\|\phi_{n}\right\|_{L^{2}(\mathbb{R})} \rightarrow 1$ as $n \rightarrow+\infty$. Then if $\tilde{V} \in \Omega_{x_{0}}^{\text {Sch }}$ with $\tilde{V} \leq 0$ we have

$$
\begin{aligned}
\left\langle\left(-\partial_{x}^{2}+V\right) \phi_{n}, \phi_{n}\right\rangle_{L^{2}(\mathbb{R})} & =\int_{\mathbb{R}}\left|\phi_{n}^{\prime}\right|^{2} d x+\int_{\mathbb{R}}\left(\tilde{V}+V_{h, q_{1}, q_{2}}\right)\left|\phi_{n}\right|^{2} d x \\
& \leq \int_{\mathbb{R}}\left|\phi_{n}^{\prime}\right|^{2} d x+\int_{\mathbb{R}} V_{h, q_{1}, q_{2}}\left|\phi_{n}\right|^{2} d x \\
& \leq \int_{-n-1}^{-n} \frac{1}{2 n} d x+\int_{n}^{n+1} \frac{1}{2 n} d x-\sum_{i=-n}^{n} \int_{\left[q_{1}, q_{2}\right]+i} \frac{h}{2 n} d x \\
& =\frac{1}{n}-\left(q_{2}-q_{1}\right) h .
\end{aligned}
$$

The assertion follows by letting $n \rightarrow+\infty$.

The next lemma is needed to construct the tower of algorithms that will compute the spectrum of elements in $\Omega_{x_{0}}^{\text {Sch }}$.

Lemma 5.4 Let $V \in \Omega_{x_{0}}^{\mathrm{Sch}}$ and for $n \in \mathbb{N}$ let $\rho_{n} \in W^{1, \infty}(\mathbb{R})$ be a periodic function such that

$$
\rho_{n}(x)= \begin{cases}0 & x \in \bigcup_{k \in \mathbb{Z}}\left(k-\frac{1}{n}, k+\frac{1}{n}\right) \\ 1 & x \in \mathbb{R} \backslash \bigcup_{k \in \mathbb{Z}}\left(k-\frac{2}{n}, k+\frac{2}{n}\right),\end{cases}
$$

then one has $\sigma\left(-\partial_{x}^{2}+\rho_{n} V\right) \rightarrow \sigma\left(-\partial_{x}^{2}+V\right)$ in Attouch-Wets distance.

Proof A Neumann series argument shows that

$$
\left(-\partial_{x}^{2}+\rho_{n} V-z\right)^{-1}-\left(-\partial_{x}^{2}+V-z\right)^{-1}=\sum_{m=1}^{\infty}\left(\left(\rho_{n}-1\right) V\left(-\partial_{x}^{2}+V-z\right)^{-1}\right)^{m}
$$

where $z \notin \mathbb{R}$ and the right hand side is defined whenever $\|\left(\rho_{n}-1\right) V\left(-\partial_{x}^{2}+V-\right.$ $z)^{-1} \|_{L^{2} \rightarrow L^{2}}<1$. We will prove that the right hand side of (5.5) converges to 0 in the norm resolvent sense. To this end let $u \in L^{2}(\mathbb{R})$ and compute

$$
\begin{aligned}
& \left\|\left(\rho_{n}-1\right) V\left(-\partial_{x}^{2}+V-z\right)^{-1} u\right\|_{L^{2}(\mathbb{R})}^{2} \\
& =\sum_{k=-\infty}^{\infty}\left\|\left(\rho_{n}-1\right) V\left(-\partial_{x}^{2}+V-z\right)^{-1} u\right\|_{L^{2}([k, k+1])}^{2}
\end{aligned}
$$




$$
\begin{aligned}
& \leq \sum_{k=-\infty}^{\infty}\left\|\left(\rho_{n}-1\right) V\right\|_{L^{2}([k, k+1])}^{2}\left\|\left(-\partial_{x}^{2}+V-z\right)^{-1} u\right\|_{L^{\infty}([k, k+1])}^{2} \\
& =\left\|\left(\rho_{n}-1\right) V\right\|_{L^{2}([0,1])}^{2} \sum_{k=-\infty}^{\infty}\left\|\left(-\partial_{x}^{2}+V-z\right)^{-1} u\right\|_{L^{\infty}([k, k+1])}^{2} \\
& \leq C\left\|\left(\rho_{n}-1\right) V\right\|_{L^{2}([0,1])}^{2} \sum_{k=-\infty}^{\infty}\left\|\left(-\partial_{x}^{2}+V-z\right)^{-1} u\right\|_{H^{1}([k, k+1])}^{2} \\
& =C\left\|\left(\rho_{n}-1\right) V\right\|_{L^{2}([0,1])}^{2}\left\|\left(-\partial_{x}^{2}+V-z\right)^{-1} u\right\|_{H^{1}(\mathbb{R})}^{2} \\
& =C\left\|\left(\rho_{n}-1\right) V\right\|_{L^{2}([0,1])}^{2}\left\|\left(-\partial_{x}^{2}+V-z\right)^{-1}\right\|_{L^{2}(\mathbb{R}) \rightarrow H^{1}(\mathbb{R})}^{2}\|u\|_{L^{2}(\mathbb{R})}^{2},
\end{aligned}
$$

where Hölder's inequality was used in the third line, periodicity of $\left(\rho_{n}-1\right) V$ was used in the fourth line and the Sobolev embedding $H^{1}(\mathbb{R}) \hookrightarrow L^{\infty}(\mathbb{R})$ was used in the fifth line. Combining Eqs. (5.5) and (5.6) we find that

$$
\begin{aligned}
& \left\|\left(-\partial_{x}^{2}+\rho_{n} V-z\right)^{-1}-\left(-\partial_{x}^{2}+V-z\right)^{-1}\right\|_{L^{2}(\mathbb{R}) \rightarrow L^{2}(\mathbb{R})} \\
& \quad \leq C \sum_{m=1}^{\infty}\left\|\left(\rho_{n}-1\right) V\right\|_{L^{2}([0,1])}^{m}\left\|\left(-\partial_{x}^{2}+V-z\right)^{-1}\right\|_{L^{2}(\mathbb{R}) \rightarrow H^{1}(\mathbb{R})}^{m},
\end{aligned}
$$

for all $n>N$ large enough such that $\left\|\left(\rho_{n}-1\right) V\right\|_{L^{2}([0,1])}<\left\|\left(-\partial_{x}^{2}+V-z\right)^{-1}\right\|_{L^{2} \rightarrow H^{1}}^{-1}$. Since $V \in L^{2}([0,1])$ we know that $\left\|\left(\rho_{n}-1\right) V\right\|_{L^{2}([0,1])} \rightarrow 0$, hence such $N$ must exist. For $n>N$ the geometric series gives

$$
\left\|\left(-\partial_{x}^{2}+\rho_{n} V-z\right)^{-1}-\left(-\partial_{x}^{2}+V-z\right)^{-1}\right\|_{L^{2}(\mathbb{R}) \rightarrow L^{2}(\mathbb{R})} \leq C \frac{q_{n}}{1-q_{n}},
$$

with $q_{n}=\left\|\left(\rho_{n}-1\right) V\right\|_{L^{2}([0,1])}\left\|\left(-\partial_{x}^{2}+V-z\right)^{-1}\right\|_{L^{2} \rightarrow H^{1}}$, which immediately implies norm resolvent convergence. Finally, an application of [30, Thms. VIII.23-24] yields the desired spectral convergence.

\subsection{Proof of Theorem 5.1}

We are now ready to prove Theorem 5.1.

Step 1: Construction of an adversarial potential $\underline{V}$. Assume for contradiction that there exists a sequence of algorithms $\left\{\Gamma_{n}\right\}_{n \in \mathbb{N}}$ such that for any $V \in \Omega_{x_{0}}^{\mathrm{Sch}}, \Gamma_{n}(V) \rightarrow$ $\sigma\left(-\partial_{x}^{2}+V\right)$ as $n \rightarrow+\infty$. We now describe a process that defines an "adversarial" potential $V$ for which the sequence $\left\{\Gamma_{n}\right\}_{n \in \mathbb{N}}$ will necessarily fail.

We begin with an example construction that illustrates how a potential can be obtained that "fools" $\Gamma_{n}$ for a single $n$. This construction is then iterated below to obtain a potential whose spectrum is not approximated by the entire sequence $\left\{\Gamma_{n}\right\}_{n \in \mathbb{N}}$. 
Sketch of $V_{1}$ :

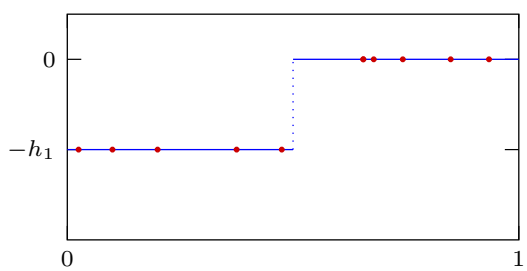

Sketch of $V_{2}$ :

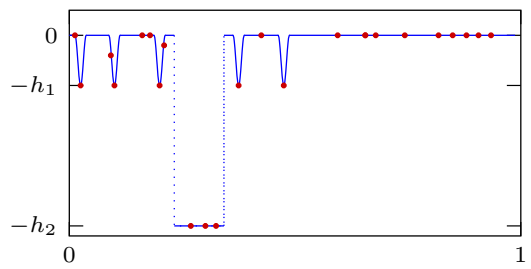

Sketch of $\tilde{V}_{1}$ :

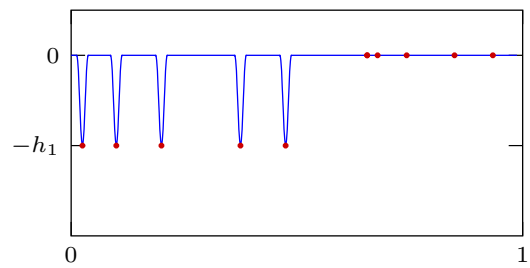

Sketch of $\tilde{V}_{2}$ :

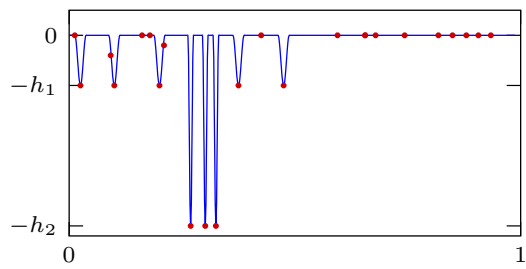

Fig. 3 Sketch the first few iterations $V_{k}, \tilde{V}_{k}$. The red dots represent the points $x_{i}$, which represent the information in $\Lambda_{\Gamma_{n}}$

For the sake of definiteness we make the arbitrary choice $x_{0}:=\frac{1}{4}$, though the proof does not depend on this choice. Moreover, we note that by Hölder's inequality one has $\|f\|_{L^{1}([0,1])} \leq\|f\|_{L^{2}([0,1])}$ for all $f \in L^{2}([0,1])$.

With the notation from Lemma 5.3, let $V_{1}$ be the periodic square well potential $V_{h_{1}, 0,1 / 2}$ with $h_{1}>0$ chosen such that inf $W\left(-\partial_{x}^{2}+V_{1}\right) \leq-1$. Because $-\partial_{x}^{2}+V$ is selfadjoint, this implies that inf $\sigma\left(-\partial_{x}^{2}+V_{1}\right) \leq-1$. Our assumptions about $\Gamma_{n}$ imply that there exists $n_{1} \in \mathbb{N}$ such that $\inf \operatorname{Re}\left(\Gamma_{n}(V)\right)<-\frac{1}{2}$ for all $n \geq n_{1}$. By the definition of an algorithm (Definition 2.3), $\Gamma_{n_{1}}(V)$ depends only on finitely many elements of $\Lambda$, i.e. finitely many point values $V\left(x_{i}\right), i \in\left\{1, \ldots, m_{n_{1}}\right\}$. We may assume without loss of generality that the sets $\left\{x_{1}, \ldots, x_{m_{n}}\right\}$ are growing with $n$.

For later reference, let $\rho$ be a smooth, compactly supported function on $\mathbb{R}$ such that

$$
\begin{aligned}
\rho(0) & =1 \\
\operatorname{supp}\left(\rho_{1}\right) & \subset\left(-\frac{1}{4}, \frac{1}{4}\right) .
\end{aligned}
$$

Next, define a new potential $\tilde{V}_{1}$ by "thinning out" $V_{1}$ around the points $x_{i}$. More concretely, let $\delta>0$ (to be determined later) and define $l_{1}:=\min \left\{\left|x_{i}-x_{j}\right| \mid i, j \in\right.$ $\left.\left\{0, \ldots, m_{n_{1}}\right\}, x_{i} \neq x_{j}\right\}$ (note that the point $x_{0}$ appears in the definition of $l_{1}$ ) and let

$$
\tilde{V}_{1}(x):=V_{1}(x) \sum_{i=1}^{m_{n_{1}}} \rho\left(\frac{x}{\delta l_{1}}-x_{i}\right) .
$$

Then by construction we have $\left\|\tilde{V}_{1}\right\|_{L^{2}([0,1])} \leq \sum_{i=1}^{m_{n_{1}}} h_{1} \sqrt{\delta l_{1}} \leq m_{n_{1}} h_{1} \sqrt{\delta}$. Next, apply Lemma 5.2 and choose $\delta$ such that inf $W\left(-\partial_{x}^{2}+\tilde{V}_{1}\right) \geq-\frac{1}{4}$ (and hence 
$\left.\inf \sigma\left(-\partial_{x}^{2}+\tilde{V}_{1}\right) \geq-\frac{1}{4}\right)$. Note that we have $\tilde{V}_{1}\left(x_{i}\right)=V_{1}\left(x_{i}\right)$ for all $i \in\left\{0, \ldots, m_{n_{1}}\right\}$. Hence by consistency of algorithms we have $\Gamma_{n_{1}}\left(\tilde{V}_{1}\right)=\Gamma_{n_{1}}\left(V_{1}\right)$. We have constructed a smooth potential such that

$$
\begin{array}{r}
\inf \operatorname{Re}\left(\Gamma_{n}\left(\tilde{V}_{1}\right)\right)<-\frac{1}{2} \\
\inf \sigma\left(-\partial_{x}^{2}+\tilde{V}_{1}\right)>-\frac{1}{4}
\end{array}
$$

and thus $d_{\mathrm{AW}}\left(\Gamma_{n_{1}}\left(\tilde{V}_{1}\right), \sigma\left(-\partial_{x}^{2}+\tilde{V}_{1}\right)\right) \geq \frac{1}{4}$. We remark that $\tilde{V}_{1} \equiv 0$ in a neighbourhood of $x_{0}$.

The constructions outlined above define an iterative process that yields a sequence of smooth potentials $\left\{V_{k}\right\}_{k \in \mathbb{N}}$ and $\left\{\tilde{V}_{k}\right\}_{k \in \mathbb{N}}$ (see Fig. 3). We outline the details below. Fix $\eta>0$ to be determined later and initialize $\tilde{V}_{0} \equiv 0$.

- Let $2 \leq k \in \mathbb{N}$ and suppose that $\tilde{V}_{k-1}$ has already been defined.

- Choose an interval $I=\left(x_{0}-\varepsilon, x_{0}+\varepsilon\right)$ on which $\tilde{V}_{k} \equiv 0$ and let $V_{k}:=\tilde{V}_{k-1}+$ $V_{h_{k}, x_{0}-\varepsilon, x_{0}+\varepsilon}$, where $h_{k}$ is chosen such that inf $W\left(-\partial_{x}^{2}+V_{k}\right) \leq-1$ (cf. Lemma 5.3).

- Choose $n_{k}$ large enough such that inf $\operatorname{Re}\left(\Gamma_{n_{k}}\left(V_{k}\right)\right)<-\frac{1}{2}$. Then $\Gamma_{n_{k}}\left(V_{k}\right)$ depends only on finitely many point values $x_{1}, \ldots, x_{m_{n_{k}}}$.

- Let $l_{k}:=\min \left\{\left|x_{i}-x_{j}\right| \mid i, j \in\left\{0, \ldots, m_{n_{k}}\right\}, x_{i} \neq x_{j}\right\}$ and define a "thinned out" potential $\tilde{V}_{k}$ by

$$
\tilde{V}_{k}(x):=\tilde{V}_{k-1}(x)+V_{h_{k}, x_{0}-\varepsilon, x_{0}+\varepsilon}(x) \sum_{i=1}^{m_{n_{k}}} \rho\left(\frac{x}{\delta l_{k}}-x_{i}\right) .
$$

Then $\left\|\tilde{V}_{k}\right\|_{L^{2}([0,1])} \leq\left\|\tilde{V}_{k-1}\right\|_{L^{2}([0,1])}+m_{n_{k}} h_{k} \sqrt{\delta}$.

- Choose $0<\delta<\left(\frac{\eta}{2^{k} m_{n_{k}} h_{k}}\right)^{2}$ such that inf $W\left(-\partial_{x}^{2}+\tilde{V}_{k}\right)>-\frac{1}{4}$.

- It follows that $\Gamma_{n_{k}}\left(\tilde{V}_{k}\right)=\Gamma_{n_{k}}\left(V_{k}\right)$ and thus $d_{\mathrm{AW}}\left(\Gamma_{n_{k}}\left(\tilde{V}_{k}\right), \sigma\left(-\partial_{x}^{2}+\tilde{V}_{k}\right)\right) \geq \frac{1}{4}$.

- Moreover, we have by construction

$$
\left\|\tilde{V}_{k}\right\|_{L^{2}([0,1])} \leq \eta \sum_{j=0}^{k} 2^{-j}
$$

and there exists an interval $\left(x_{0}-\varepsilon^{\prime}, x_{0}+\varepsilon^{\prime}\right)$ on which $\tilde{V}_{k} \equiv 0$.

This process defines a sequence of potentials $\left\{\tilde{V}_{k}\right\}_{k \in \mathbb{N}}$ such that for a subsequence $\left\{n_{k}\right\}_{k \in \mathbb{N}}$ one has

$$
\liminf _{k \rightarrow+\infty} d_{\mathrm{AW}}\left(\Gamma_{n_{k}}\left(\tilde{V}_{k}\right), \sigma\left(-\partial_{x}^{2}+\tilde{V}_{k}\right)\right) \geq \frac{1}{4}
$$


Next we show that the sequence $\tilde{V}_{k}$ converges pointwise to a function $V^{\eta} \in L^{2}([0,1])$, which is smooth on $[0,1] \backslash\left\{x_{0}\right\}$. By construction, for every $\varepsilon>0$, sequence $\left\{\left.\tilde{V}_{k}\right|_{[0,1] \backslash\left(x_{0}-\varepsilon, x_{0}+\varepsilon\right)}\right\}_{k \in \mathbb{N}}$ is eventually constant. Combined with the fact that $\tilde{V}_{k}\left(x_{0}\right)=$ 0 for all $k$, this implies that $\left\{\tilde{V}_{k}\right\}_{k \in \mathbb{N}}$ converges pointwise to a function $V^{\eta}$ on $[0,1]$. Because for every $\varepsilon>0$ there exists $k \in \mathbb{N}$ such that $\left.V^{\eta}\right|_{[0,1] \backslash\left(x_{0}-\varepsilon, x_{0}+\varepsilon\right)}=$ $\left.\tilde{V}_{k}\right|_{[0,1] \backslash\left(x_{0}-\varepsilon, x_{0}+\varepsilon\right)}$, we have that $V^{\eta}$ is smooth on $[0,1] \backslash\left\{x_{0}\right\}$. Finally, by $(5.7)$ for any $\varepsilon>0$ there exists $k \in \mathbb{N}$ such that

$$
\begin{aligned}
\left\|V^{\eta}\right\|_{L^{2}\left([0,1] \backslash\left(x_{0}-\varepsilon, x_{0}+\varepsilon\right)\right)} & =\left\|\tilde{V}_{k}\right\|_{L^{2}\left([0,1] \backslash\left(x_{0}-\varepsilon, x_{0}+\varepsilon\right)\right)} \\
& \leq \eta \sum_{j=0}^{k} 2^{-j} \\
& \leq 2 \eta .
\end{aligned}
$$

Letting $\varepsilon \rightarrow 0$ we conclude by monotone convergence that $V^{\eta} \in L^{2}([0,1])$ and consequently $V^{\eta} \in \Omega_{x_{0}}^{\text {Sch }}$. Moreover, the inequality $\left\|V^{\eta}\right\|_{L^{2}([0,1])} \leq 2 \eta$ allows us to use Lemma 5.2 and choose $\eta>0$ small enough that

$$
\inf W\left(-\partial_{x}^{2}+V^{\eta}\right)=\inf \sigma\left(-\partial_{x}^{2}+V^{\eta}\right) \geq-\frac{1}{4}
$$

To conclude the proof we note that for any $k \in \mathbb{N}$ we have $V^{\eta}\left(x_{i}\right)=\tilde{V}_{k}\left(x_{i}\right)$ for all $i \in\left\{1, \ldots, m_{n_{k}}\right\}$ and by consistency of algorithms we have

$$
\Gamma_{n_{k}}\left(V^{\eta}\right)=\Gamma_{n_{k}}\left(\tilde{V}_{k}\right)
$$

and thus inf $\operatorname{Re}\left(\Gamma_{n_{k}}\left(V^{\eta}\right)\right) \leq-\frac{1}{2}$. Combining eqs. (5.8), (5.9) and (5.10) we conclude that

$$
\liminf _{k \rightarrow+\infty} d_{\mathrm{AW}}\left(\Gamma_{n_{k}}\left(V^{\eta}\right), \sigma\left(-\partial_{x}^{2}+V^{\eta}\right)\right) \geq \frac{1}{4} .
$$

Consequently, the sequence $\Gamma_{n}\left(V^{\eta}\right)$ cannot converge to $\sigma\left(-\partial_{x}^{2}+V^{\eta}\right)$ and the desired contradiction follows, proving that $\operatorname{SCI}\left(\Omega_{x_{0}}^{\mathrm{Sch}}\right) \geq 2$.

Step 2: Construction of tower of algorithms. We conclude the proof of Theorem 5.1 by showing $\operatorname{SCI}\left(\Omega_{x_{0}}^{\mathrm{Sch}}\right) \leq 2$. To this end, choose a function $\rho_{n}$ as in (5.4) change this, if we change the $\rho_{n}$ above, whose values are explicitly computable (e.g. piecewise linear) and define the mapping

$$
\begin{array}{r}
\Gamma_{m, n}: \Omega_{x_{0}}^{\mathrm{Sch}} \rightarrow \mathcal{M} \\
\Gamma_{m, n}(V)=\Gamma_{m}\left(\rho_{n} V\right),
\end{array}
$$

where $\Gamma_{m}$ denotes the algorithm from Definition 4.13. Note that $\Gamma_{m}\left(\rho_{n} V\right)$ is welldefined because $\rho_{n} V \in W^{1, \infty}(\mathbb{R})$ for every $n \in \mathbb{N}$. Applying Theorem 1.2 and Lemma 5.4 we immediately find 


$$
\begin{aligned}
\lim _{n \rightarrow+\infty} \lim _{m \rightarrow+\infty} \Gamma_{m, n}(V) & =\lim _{n \rightarrow+\infty} \lim _{m \rightarrow+\infty} \Gamma_{m}\left(\rho_{n} V\right) \\
& =\lim _{n \rightarrow+\infty} \sigma\left(-\partial_{x}^{2}+\rho_{n} V\right) \\
& =\sigma\left(-\partial_{x}^{2}+V\right),
\end{aligned}
$$

where all limits are taken in Attouch-Wets distance. This completes the proof.

Note that the two limits in Step 2 above cannot be swapped, because it is unclear how $\Gamma_{m}\left(\rho_{n} V\right)$ behaves when $\rho_{n} V$ converges to a non-smooth function.

\section{Numerical results}

To illustrate our abstract results, we implemented a version of the algorithm from Definition 4.13 in one dimension in Matlab. In this section we show the results of this implementation and compare them against known abstract and numerical results.

In order to obtain an implementation with adequate performance, we fixed a box $Q$ in the complex plane and then computed the quantity

$$
\bigcup_{\theta \in \frac{1}{N} \mathbb{Z} \cap[0,2 \pi]}\left\{z \in \frac{1}{N}(\mathbb{Z}+1 \mathbb{Z}) \cap Q|| \operatorname{det}\left(I-P_{N} K_{n}^{\mathrm{appr}}(z, \theta) P_{N}\right) \mid \leq C\right\},
$$

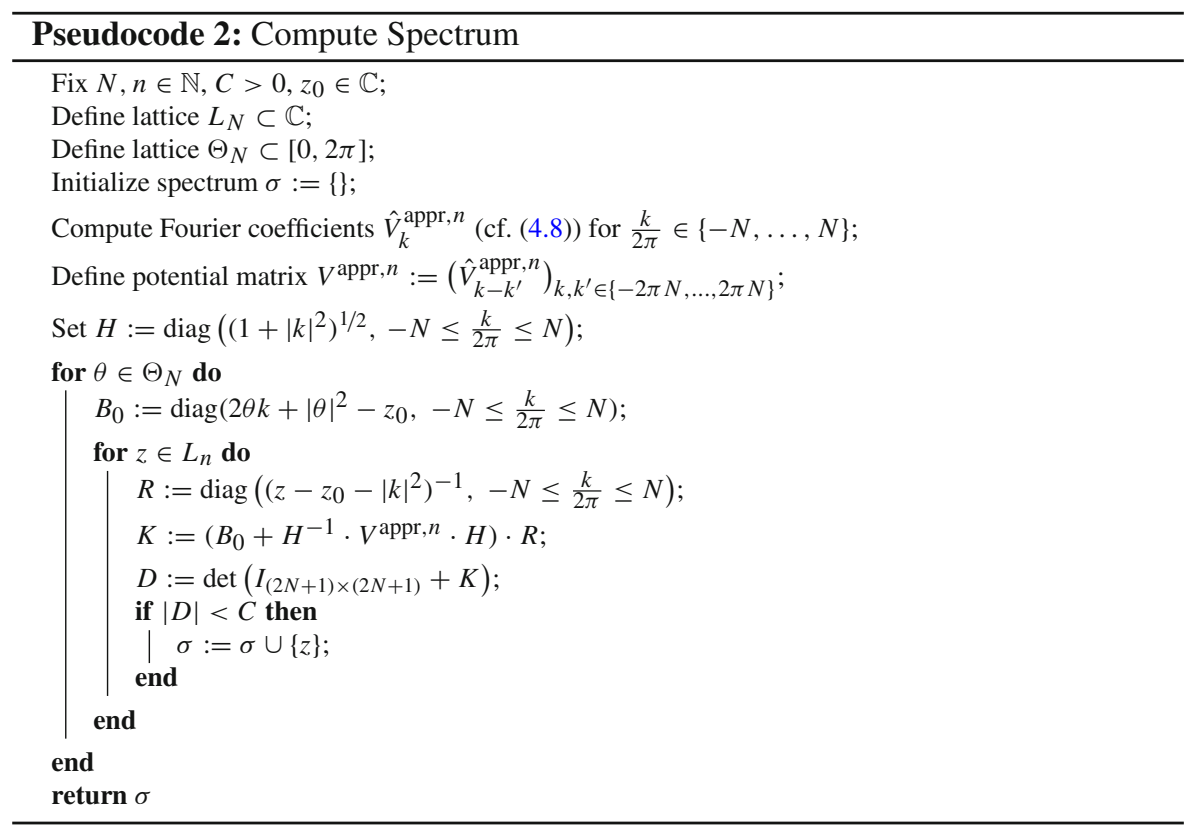



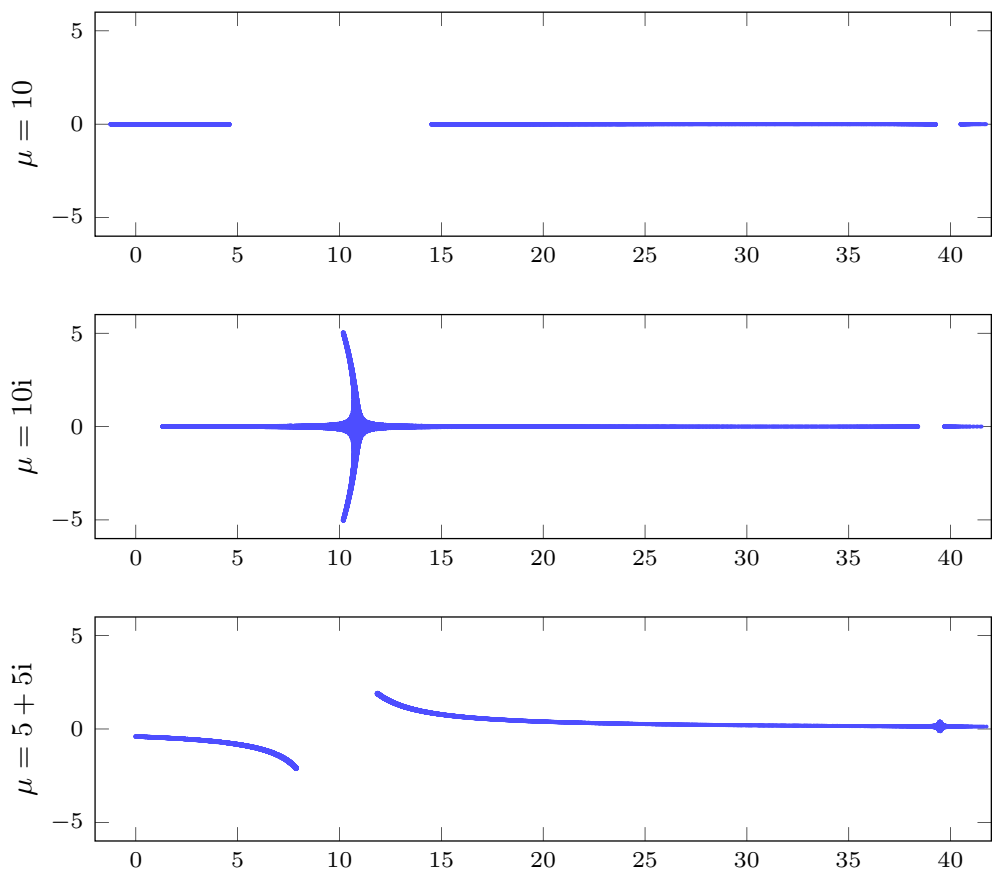

Fig. 4 Spectral approximation in $\mathbb{C}$ for the Mathieu operator for $\mu \in\{10,101,5+51\}$. Parameter values: $N=200, z_{0}=45, n=50, C=2 \cdot 10^{-5}$

where the numbers $N, n, C$ were treated as independent parameters. Moreover, the spectral shift, which was chosen to be 1 in (4.1) and 2 in (4.17) can be fixed to be any point $z_{0}$ outside the box $Q$. The routine is illustrated in Pseudocode 2 .

The actual Matlab implementation of Pseudocode 2 is available on https://github. com/frank-roesler/PeriodicSpectra.

\subsection{Implementation in one dimension. The Mathieu equation}

We consider the Mathieu equation

$$
-u^{\prime \prime}(x)+\mu \cos (2 \pi x) u(x)=\lambda u(x)
$$

where $\mu \in \mathbb{C}$ is a constant and $\lambda$ denotes the spectral parameter. This equation was first studied in [25] in the context of vibrating membranes and has been studied extensively since (see [26, Ch. 5] for a discussion). Figure 4 shows the output of our implementation for various values of $\mu$.

In the case of a real-valued potential (top panel of Fig. 4) our algorithm produces the expected band-gap structure, with one gap showing around $\lambda=10$ and another around $\lambda=40$. In the case of purely imaginary $\mu$, the theory of $\mathcal{P} \mathcal{T}$-symmetric operators (i.e. operators that are invariant under simultaneous parity and time reversal) can be used to prove abstract results about the possible shape of the spectrum [34]. A comparison 


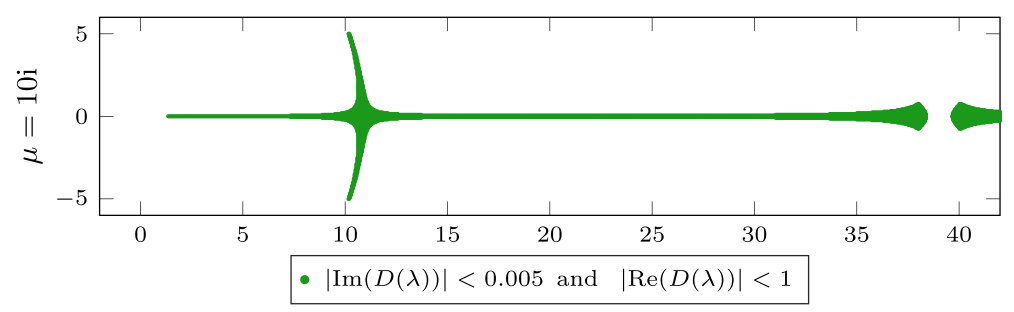

Fig. 5 Spectral approximation with discriminant method for $\mu=101$

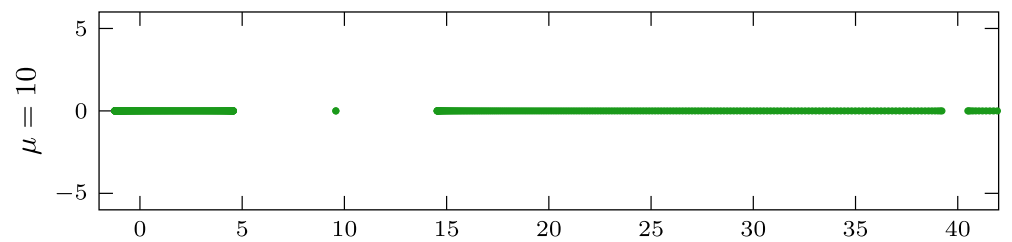

Fig. 6 Spectral approximation from finite difference scheme on truncated domain $[-100.7,100.7]$. The spurious eigenvalue in the gap for $\mu=10$ can appear for arbitrarily fine discretization

between the middle panel of Fig. 4 and [34, Fig. 2] shows agreement between the theoretical results and the output of our algorithm. Finally, the bottom panel in Fig. 4 shows the output when $\mu$ has both a real and an imaginary part and the $\mathcal{P} \mathcal{T}$ symmetry is broken.

To further validate our results, let us focus on the $\mathcal{P} \mathcal{T}$ symmetric case $\left(\mu=10_{1}\right)$ and compare them to existing results available in one dimension. Let $\phi_{1, \lambda}, \phi_{2, \lambda}$ be two classical solutions of $(6.1)$ on $(0,1)$ with initial conditions $\phi_{1, \lambda}(0)=1, \phi_{1, \lambda}^{\prime}(0)=0$ and $\phi_{2, \lambda}(0)=0, \phi_{2, \lambda}^{\prime}(0)=1$. Then, the Hill Discriminant is defined by

$$
D(\lambda):=\frac{1}{2}\left(\phi_{1, \lambda}(1)+\phi_{2, \lambda}^{\prime}\right.
$$

and one can show (cf. [15]) that $\lambda$ is in the spectrum of (6.1) if and only if $-1 \leq D(\lambda) \leq$ 1. Figure 5 shows the points in $\mathbb{C}$ satisfying a softened version of this discriminant inequality, computed from a Runge-Kutta approximation of $\phi_{1, \lambda}, \phi_{2, \lambda}$. A comparison between Figs. 5 and 4 shows good agreement.

Finally, we note that our method is naturally immune to the common problem of spectral pollution. By definition, spectral pollution occurs when there exist sequences $z_{n} \in \Gamma_{n}(V)$ such that $\left\{z_{n}\right\}_{n \in \mathbb{N}}$ has an accumulation point outside $\sigma(-\Delta+V)$. This effect appears in the approximation of Mathieu's equation if a naive finite difference scheme is used on a truncated domain (see Fig. 6). For arbitrarily fine discretization and arbitrarily large truncated domain, the method is sensitive to small perturbations of the domain and can yield a set which is far from the correct spectrum. 


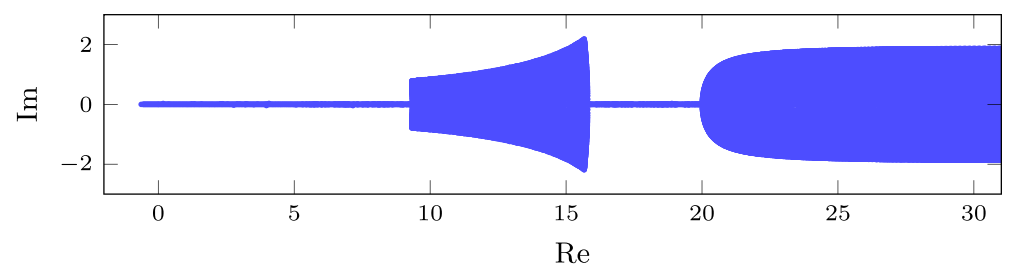

Fig. 7 Output of the algorithm in 2 dimensions for $\frac{k}{2 \pi} \in\{-10, \ldots, 10\}$

\subsection{Implementation in two dimensions}

In principle, the algorithm given by Pseudocode 2 can be implemented in any space dimension. However, in $d$ dimensions the size of the matrix $V^{\text {appr, } n}$ grows like $n^{d}$ and the computation quickly becomes unfeasible.

In the case $d=2$ an algorithm based on Pseudocode 2 can still be implemented, with certain modifications. A full search of the lattice $L_{N}$ is not feasible anymore. Instead we implemented an algorithm that finds zeros of $D$ successively: Having computed a zero $z_{0}$, increment $\theta$ and choose a number of points $\left\{w_{1}, \ldots, w_{k}\right\}$ and for each $i \in\{1, \ldots, k\}$ minimize $D$ using $w_{i}$ as starting point. Increment $\theta$ again and continue. A Matlab implementation of this idea is on https://github.com/frankroesler/PeriodicSpectra2d.

Figure 7 shows the output of our algorithm for the potential

$$
V(x, y)=9 \sin (2 \pi y)+101 \cos (2 \pi x) \sin (2 \pi y) .
$$

Note that $V$ has a finite Fourier expansion. This makes the matrix $V^{\text {appr, } n}$ sparse and speeds up the computation. More generic potentials can only be computed with significantly reduced accuracy.

Open Access This article is licensed under a Creative Commons Attribution 4.0 International License, which permits use, sharing, adaptation, distribution and reproduction in any medium or format, as long as you give appropriate credit to the original author(s) and the source, provide a link to the Creative Commons licence, and indicate if changes were made. The images or other third party material in this article are included in the article's Creative Commons licence, unless indicated otherwise in a credit line to the material. If material is not included in the article's Creative Commons licence and your intended use is not permitted by statutory regulation or exceeds the permitted use, you will need to obtain permission directly from the copyright holder. To view a copy of this licence, visit http://creativecommons.org/licenses/by/4.0/.

\section{Appendix A. The Hausdorff and Attouch-Wets distances}

Since the Attouch-Wets distance is not well-known, we provide the basic definition here, and use the opportunity to remind the reader of the Hausdorff distance as well. For further information we refer to [3]. 
Definition A.1 (Hausdorff distance for subsets of $\mathbb{R}^{d}$ ) Let $A, B \subset \mathbb{R}^{d}$ be two nonempty bounded sets. Their Hausdorff distance is

$$
\begin{aligned}
d_{\mathrm{H}}(A, B) & =\max \left\{\sup _{a \in A} \inf _{b \in B}|a-b|, \sup _{b \in B} \inf _{a \in A}|a-b|\right\} \\
& =\sup _{p \in \mathbb{R}^{d}}\left|\inf _{a \in A}\right| a-p\left|-\inf _{b \in B}\right| b-p|| .
\end{aligned}
$$

Definition A.2 (Attouch-Wets distance for subsets of $\mathbb{R}^{d}$ ) Let $A, B \subset \mathbb{R}^{d}$ be two nonempty (possibly unbounded) sets. Their Attouch-Wets distance is

$$
d_{\mathrm{AW}}(A, B)=\sum_{n=1}^{\infty} 2^{-n} \min \left\{1, \sup _{p \in \mathbb{R}^{d},|p|<n}\left|\inf _{a \in A}\right| a-p\left|-\inf _{b \in B}\right| b-p||\right\} .
$$

Note that if $A, B$ are bounded, then $d_{\mathrm{AW}}$ and $d_{\mathrm{H}}$ are equivalent. Furthermore, it can be shown (cf. [3, Ch. 3]) that

$$
d_{\mathrm{H}}\left(A_{n} \cap B, A \cap B\right) \rightarrow 0 \text { for all } B \subset \mathbb{R}^{d} \text { compact } \Rightarrow d_{\mathrm{AW}}\left(A_{n}, A\right) \rightarrow 0 .
$$

\section{Appendix B. Floquet-Bloch transform}

The Floquet-Bloch transform for Schrödinger operators with periodic potentials is well-studied. The following lemma is a collection of results in [29] (below, $\mathcal{S}\left(\mathbb{R}^{d}\right)$ denotes the Schwartz space of rapidly decaying functions).

Lemma B.1 ( $\left[29\right.$, Ch. XIII.16]) For $f \in \mathcal{S}\left(\mathbb{R}^{d}\right)$ and $\theta \in[0,2 \pi]^{d}$, define the map

$$
\left(\mathcal{U}_{\theta} f\right)(x):=(2 \pi)^{-\frac{d}{2}} \sum_{n \in \mathbb{Z}^{d}} f(x+n) e^{1 \theta \cdot(x+n)} .
$$

Then the following hold.

(i) $\mathcal{U}_{\theta}$ extends uniquely to a bounded operator on $L^{2}\left(\mathbb{R}^{d}\right)$;

(ii) For any $f \in L^{2}\left(\mathbb{R}^{d}\right), \mathcal{U}_{\theta} f$ is 1-periodic;

(iii) The map

$$
\begin{aligned}
\mathcal{U}: L^{2}\left(\mathbb{R}^{d}\right) & \rightarrow \int_{[0,2 \pi]^{d}}^{\oplus} L^{2}\left((0,1)^{d}\right) d \theta \\
f & \mapsto\left(\mathcal{U}_{\theta} f\right)_{\theta \in[0,2 \pi]^{d}}
\end{aligned}
$$

is unitary;

(iv) The inverse $\mathcal{U}^{-1}$ is given by

$$
\left(\mathcal{U}^{-1} g\right)(x+n)=(2 \pi)^{-\frac{d}{2}} \int_{[0,2 \pi]^{d}} g(x, \theta) e^{-1 n \theta} d \theta
$$


(v) For $H=-\Delta+V, V \in \Omega_{p}$ one has

$$
\mathcal{U} H \mathcal{U}^{-1}=\int_{[0,2 \pi]^{d}}^{\oplus} H(\theta) d \theta
$$

where

$$
\begin{aligned}
H(\theta) & =-(\nabla+1 \theta)^{2}+V, \\
\operatorname{dom}(H(\theta)) & =H_{\text {per }}^{2}\left((0,1)^{d}\right) \quad \forall \theta \in[0,2 \pi]^{d} .
\end{aligned}
$$

Moreover, the map $\theta \mapsto H(\theta)$ is analytic and

$$
\sigma(H)=\bigcup_{\theta \in[0,2 \pi]^{d}} \sigma(H(\theta)) .
$$

The spectral identity (B.2) follows from the unitarity of $\mathcal{U}$ and a straightforward calculation, noting that $\bigcup_{\theta \in[0,2 \pi]^{d}} \sigma(H(\theta))$ is closed by analyticity and periodicity in $\theta$.

\section{Appendix C. Proof of proposition 4.9}

(i) Let $z_{N} \in \Gamma_{N}^{z_{0}}(V)$ and assume that $z_{N} \rightarrow z$ for some $z \in \mathbb{C}$. We need to show that $z \in \sigma(-\Delta+V)$. Since $z_{N} \in \Gamma_{N}^{z_{0}}(V)$, we have $\operatorname{det}_{\lceil p\rceil}\left(I-P_{N} K_{n(N)}^{\text {appr }}\left(z, \theta_{i_{N}}\right) P_{N}\right) \rightarrow 0$ for some sequence $\left\{\theta_{i_{N}}\right\}_{N \in \mathbb{N}} \subset[0,2 \pi]^{d}$. Then there exists a convergent subsequence (again denoted by $\theta_{i_{N}}$ ) converging to some some $\theta \in[0,2 \pi]^{d}$. We first note that due to Theorem 4.7 we have the $N$-independent determinant error bound

$$
\begin{gathered}
\left|\operatorname{det}_{\lceil p\rceil}\left(I-K\left(z_{N}, \theta_{i_{N}}\right)\right)-\operatorname{det}_{\lceil p\urcorner}\left(I-P_{N} K_{n(N)}^{\mathrm{appr}}\left(z_{N}, \theta_{i_{N}}\right) P_{N}\right)\right| \\
\leq\left\|K\left(z_{N}, \theta_{i_{N}}\right)-P_{N} K_{n(N)}^{\mathrm{appr}}\left(z_{N}, \theta_{i_{N}}\right) P_{N}\right\|_{\mathcal{C}_{\lceil p\rceil}} \times \\
e^{c_{\lceil p\rceil}\left(1+\left\|K\left(z_{N}, \theta_{i_{N}}\right)\right\|_{\mathcal{C}_{\lceil p\rceil}}+\left\|K_{n(N)}^{\mathrm{appr}}\left(z_{N}, \theta_{i_{N}}\right)\right\|_{\left.\mathcal{C}_{\lceil p\rceil}\right)},\right.}
\end{gathered}
$$

(note that $\operatorname{det}_{\lceil p\rceil}\left(I-K\left(z_{N}, \theta_{i_{N}}\right)\right.$ ) is well-defined by Lemma 4.2 because $p>d$ ). We note that the exponential factor in the last line is uniformly bounded in $N$ by some explicit constant $c_{\text {exp }}$ (cf. Lemmas 4.2 and 4.6). Using Lemma 4.6 with our choice $n(N)=N^{\lceil\alpha\rceil}$ and $s=p$, we obtain

$$
\begin{aligned}
\mid \operatorname{det}_{\lceil p\urcorner}(I- & \left.K\left(z_{N}, \theta_{i_{N}}\right)\right)-\operatorname{det}_{\lceil p\rceil}\left(I-P_{N} K_{n(N)}^{\mathrm{appr}}\left(z_{N}, \theta_{i_{N}}\right) P_{N}\right) \mid \\
\leq & c_{\exp } C_{z_{N}}\left(C_{p, d}^{1}\left|\theta_{i_{N}}\right| N^{\frac{1}{p}-\frac{1}{d}}+C_{p, p, d}^{2} N^{\frac{1}{p}-\frac{1}{d}}\|V\|_{W^{1, p}}\right. \\
& \left.+C_{p, d}^{3} N^{\frac{1}{p}-\frac{1}{d}}\left(\left.|| \theta_{i_{N}}\right|^{2}-1 \mid+\|V\|_{L^{\infty}}\right)\right),
\end{aligned}
$$


Note that each term on the right hand side has a negative power of $N$ and therefore tends to 0 as $N \rightarrow+\infty$. Note further that $C_{z_{N}}=\sup _{j \in \mathbb{N}}\left|1-\frac{z_{N}-1}{\left|k_{j}\right|^{2}}\right|^{-1}$ remains bounded as $N \rightarrow+\infty$ by our assumption that $z \notin \bigcup_{j}\left(1+\left|k_{j}\right|^{2}\right)$. Therefore we have

$$
\left|\operatorname{det}_{\lceil p\rceil}\left(I-K\left(z_{N}, \theta_{i_{N}}\right)\right)-\operatorname{det}_{\lceil p\urcorner}\left(I-P_{N} K_{n(N)}^{\mathrm{appr}}\left(z_{N}, \theta_{i_{N}}\right) P_{N}\right)\right| \rightarrow 0 \quad \text { as } N \rightarrow+\infty .
$$

To conclude the proof of (i), if we note that continuity in $(z, \theta)$ and periodicity in $\theta$ imply

$$
\begin{aligned}
\left|\operatorname{det}_{\lceil p\rceil}(I-K(z, \theta))\right|= & \lim _{N \rightarrow+\infty}\left|\operatorname{det}_{\lceil p\rceil}\left(I-K\left(z_{N}, \theta_{i_{N}}\right)\right)\right| \\
\leq & \lim _{N \rightarrow+\infty}\left|\operatorname{det}_{\lceil p\rceil}\left(I-P_{N} K_{n(N)}^{\mathrm{appr}}\left(z_{N}, \theta_{i_{N}}\right) P_{N}\right)\right| \\
& \quad+\lim _{N \rightarrow+\infty} \mid \operatorname{det}_{\lceil p\rceil}\left(I-K\left(z_{N}, \theta_{i_{N}}\right)\right) \\
& -\operatorname{det}_{\lceil p\urcorner}\left(I-P_{N} K_{n(N)}^{\mathrm{appr}}\left(z_{N}, \theta_{i_{N}}\right) P_{N}\right) \mid \\
= & 0 .
\end{aligned}
$$

Hence we have $1 \in \sigma(K(z, \theta))$ and thus $z \in \sigma(-\Delta+V)$.

(ii) Conversely, denote $H:=-\Delta+V$ and let $z \in \sigma(H) \cap Q_{z_{0}} \backslash \bigcup_{j}\left(1+\left|k_{j}\right|^{2}\right)$. We need to show that there exists a sequence $z_{N} \in \Gamma_{N}^{z_{0}}(V)$ such that $z_{N} \rightarrow z$. In fact, let $z_{N} \in L_{N}$ be any sequence with $\left|z-z_{N}\right|<\frac{1}{N}$ (such a sequence exists by the definition of $\left.L_{N}\right)$. Since $z \in \sigma(H)$ there exists $\theta \in[0,2 \pi]^{d}$ with $z \in \sigma(H(\theta))$, cf. Lemma B.1. Consequently

$$
\operatorname{det}_{\lceil p\rceil}(I-K(z, \theta))=0 .
$$

Consider some sequence $\theta_{N} \in \Theta_{N}$ with $\left|\theta_{N}-\theta\right|<\frac{1}{N}$, the existence of which is guaranteed by the definition of $\Theta_{N}$. By Lemma 4.3 and Theorem 4.7 there exists $C>0$ with

$$
\left|\operatorname{det}_{\lceil p\urcorner}\left(I-K\left(z_{N}, \theta_{N}\right)\right)\right| \leq C\left(\left|z-z_{N}\right|+\left|\theta-\theta_{N}\right|\right) \leq \frac{2 C}{N} .
$$

Thus, using (C.2) and the main error estimate (C.1), we obtain

$$
\begin{aligned}
& \left|\operatorname{det}_{\lceil p\urcorner}\left(I-P_{N} K_{n(N)}^{\mathrm{appr}}\left(z_{N}, \theta_{N}\right) P_{N}\right)\right| \\
& \leq\left|\operatorname{det}_{\lceil p\urcorner}\left(I-K\left(z_{N}, \theta_{N}\right)\right)\right| \\
& +\mid \operatorname{det}_{\lceil p\urcorner}\left(I-P_{N} K_{n(N)}^{\mathrm{appr}}\left(z_{N}, \theta_{N}\right) P_{N}\right) \\
& \quad-\operatorname{det}_{\lceil p\urcorner}\left(I-K\left(z_{N}, \theta_{N}\right)\right) \mid
\end{aligned}
$$




$$
\begin{aligned}
\leq & \frac{2 C}{N}+c_{\exp } C_{z_{N}} N^{\frac{1}{p}-\frac{1}{d}} \times \\
& \left(C_{p, d}^{1}\left|\theta_{N}\right|+C_{p, d}^{2}\|V\|_{W^{1, p}\left((0,1)^{d}\right)}\right. \\
& \left.+C_{p, d}^{3}\left(\left.|| \theta_{N}\right|^{2}-1 \mid+\|V\|_{L^{\infty}\left((0,1)^{d}\right)}\right)\right) .
\end{aligned}
$$

To keep the notation simple, we collect all constants independent of $N$ into a single constant $G=G(p, d, V)$ (note that $\left|\theta_{N}\right| \leq 2 \pi \sqrt{d}$ for all $N$ ). This gives

$$
\left|\operatorname{det}_{\lceil p\urcorner}\left(I-P_{N} K_{n(N)}^{\mathrm{appr}}\left(z_{N}, \theta_{N}\right) P_{N}\right)\right| \leq \frac{2 C}{N}+c_{\exp } G C_{z_{N}} N^{\frac{1}{p}-\frac{1}{d}} \leq N^{-\left(\frac{1}{2 d}-\frac{1}{2 p}\right)},
$$

where the last line holds for $N$ large enough (note that $\frac{1}{2 d}-\frac{1}{2 p}>0$ by our assumption $p>d$ and that $C_{z_{N}}$ remains bounded as $N \rightarrow+\infty$ by our assumptions on $z$ ). Comparing (C.3) to (4.15), we see that $z_{N} \in \Gamma_{N}^{z_{0}}(V)$ for $N$ large enough.

(iii) The proof is similar to that of (ii). Let $z \in\left(\sigma(-\Delta+V) \cap Q_{z_{0}}\right) \backslash B_{\delta}\left(\bigcup_{j}(1+\right.$ $\left.\left.\left|k_{j}\right|^{2}\right)\right)$ be an arbitrary point and let $z_{N} \in L_{N}$ be any sequence with $\left|z-z_{N}\right|<\frac{1}{N}$. Since $z \in \sigma(H)$ there exists $\theta \in[0,2 \pi]^{d}$ with $z \in \sigma(H(\theta))$, cf. Lemma B.1. Consequently

$$
\operatorname{det}_{\lceil p\rceil}(I-K(z, \theta))=0 .
$$

Consider some sequence $\theta_{N} \in \Theta_{N}$ with $\left|\theta_{N}-\theta\right|<\frac{1}{N}$, the existence of which is guaranteed by the definition of $\Theta_{N}$. By Corollary 4.4 and Theorem 4.7 this implies

$$
\begin{aligned}
\left|\operatorname{det}_{\lceil p\urcorner}\left(I-K\left(z_{N}, \theta_{N}\right)\right)\right| & \leq C_{\delta, z_{0}}^{\operatorname{Lip}}\left(1+\|V\|_{W^{1, p}\left((0,1)^{d}\right)}\right)\left(\left|\theta-\theta_{N}\right|+\left|z-z_{N}\right|\right) \\
& \leq 2 N^{-1} C_{\delta, z_{0}}^{\operatorname{Lip}}\left(1+\|V\|_{W^{1, p}\left((0,1)^{d}\right)}\right)
\end{aligned}
$$

where $C_{\delta, z_{0}}^{\mathrm{Lip}}=c_{\exp } \delta^{-2} 48\left(\left|z_{0}\right|+1\right)^{2} \frac{s d}{s-d} \frac{3 p-d}{p-d}$. Turning to the finite approximation $K_{n}^{\text {appr }}$, this gives

$$
\begin{aligned}
& \left|\operatorname{det}_{\lceil p\urcorner}\left(I-P_{N} K_{n(N)}^{\mathrm{appr}}\left(z_{N}, \theta_{N}\right) P_{N}\left(z_{N}, \theta_{N}\right)\right)\right| \\
& \quad \leq\left|\operatorname{det}_{\lceil p\urcorner}\left(I-K\left(z_{N}, \theta_{N}\right)\right)\right| \\
& \quad+\left|\operatorname{det}_{\lceil p\urcorner}\left(I-K\left(z_{N}, \theta_{N}\right)\right)-\operatorname{det}_{\lceil p\urcorner}\left(I-K^{\mathrm{appr}}\left(z_{N}, \theta_{N}\right)\right)\right| \\
& \quad \leq 2 N^{-1} C_{\delta, z_{0}}^{\mathrm{Lip}}\left(1+\|V\|_{W^{1, p}\left((0,1)^{d}\right)}\right)+G C_{z_{N}} N^{\frac{1}{p}-\frac{1}{d}} \\
& \quad \leq 2 N^{-1} C_{\delta, z_{0}}^{\mathrm{Lip}}\left(1+\|V\|_{W^{1, p}\left((0,1)^{d}\right)}\right)+G\left(\left|z_{0}\right|+1\right) \delta^{-1} N^{\frac{1}{p}-\frac{1}{d}},
\end{aligned}
$$

where $G$ denotes the explicit and computable constant introduced in step (ii) above and we have used the bound $C_{z_{N}} \leq\left(\left|z_{0}\right|+1\right) \delta^{-1}$. The condition $z_{N} \in \Gamma_{N}(V)$ is thus implied by 
$2 N^{-1} C_{\delta, z_{0}}^{\operatorname{Lip}}\left(1+\|V\|_{W^{1, p}\left((0,1)^{d}\right)}\right)+G\left(\left|z_{0}\right|+1\right) \delta^{-1} N^{\frac{1}{p}-\frac{1}{d}}<N^{-(1 / 2 d-1 / 2 p)}$,

which immediately implies the assertion, noting that $\left|z-z_{N}\right|<\frac{1}{N}<\varepsilon$ as soon as $N>\varepsilon^{-1}$.

\section{References}

1. Avila, A., Jitomirskaya, S.: The Ten Martini Problem. Ann. Math., 303-342 (2009)

2. Becker, S., Hansen, A.C.: Computing solutions of Schrödinger equations on unbounded domains-On the brink of numerical algorithms. arXiv: 2010.16347 e-prints (2020)

3. Beer, G.: Topologies on closed and closed convex sets. Mathematics and its Applications, vol. 268. Kluwer Academic Publishers Group, Dordrecht (1993)

4. Ben-Artzi, J., Colbrook, M.J., Hansen, A.C., Nevanlinna, O., Seidel, M.: Computing spectra—On the solvability complexity index hierarchy and towers of algorithms. arXiv: 1508.03280 e-prints (2015)

5. Ben-Artzi, J., Hansen, A.C., Nevanlinna, O., Seidel, M.: New barriers in complexity theory: on the solvability complexity index and the towers of algorithms. Comptes Rendus Math. 353(10), 931-936 (2015)

6. Ben-Artzi, J., Marletta, M., Rösler, F.: Computing scattering resonances. arXiv e-prints (2020)

7. Ben-Artzi, J., Marletta, M., Rösler, F.: Computing the sound of the sea in a seashell. Found. Comput. Math. (2020)

8. Birman, M.V.: On the spectrum of singular boundary-value problems. Math. Sb. 55(97), 125-174 (1961)

9. Brezis, H.: Functional analysis, Sobolev spaces and partial differential equations. Universitext, Springer, New York (2011)

10. Coddington, E.A., Levinson, N.: Theory of ordinary differential equations. McGraw-Hill Book Company Inc, New York-Toronto-London (1955)

11. Colbrook, M.J.: On the computation of geometric features of spectra of linear operators on Hilbert spaces. arXiv: 1908.09598 e-prints (2019)

12. Colbrook, M.J., Hansen, A.C.: The foundations of spectral computations via the Solvability Complexity Index hierarchy: part I. arXiv: 1908.09592 e-print (2019)

13. Colbrook, M.J., Roman, B., Hansen, A.C.: How to compute spectra with error control. Phys. Rev. Lett. 122(25), 250201 (2019)

14. Dunford, N., Schwartz, J.T.: Linear operators. Part II. Wiley Classics Library. John Wiley \& Sons, Inc., New York (1988). Spectral theory. Selfadjoint operators in Hilbert space, with the assistance of Bade, W.G., and Bartle, R.G., Reprint of the 1963 original. A Wiley-Interscience Publication

15. Eastham, M.S.P.: The spectral theory of periodic differential equations. Texts in Mathematics (Edinburgh). Scottish Academic Press, Edinburgh; Hafner Press, New York (1973)

16. Figotin, A., Kuchment, P.: Band-gap structure of the spectrum of periodic Maxwell operators. J. Stat. Phys. 74(1-2), 447-455 (1994)

17. Figotin, A., Kuchment, P.: Band-gap structure of spectra of periodic dielectric and acoustic media. I. Scalar model. SIAM J. Appl. Math. 56(1), 68-88 (1996)

18. Figotin, A., Kuchment, P.: Band-gap structure of spectra of periodic dielectric and acoustic media. II. Two-dimensional photonic crystals. SIAM J. Appl. Math. 56(6), 1561-1620 (1996)

19. Giani, S., Graham, I.G.: Adaptive finite element methods for computing band gaps in photonic crystals. Numer. Math. 121(1), 31-64 (2012)

20. Gohberg, I., Goldberg, S., Kaashoek, M. A.: Laurent and Toeplitz Operators. In Basic Classes of Linear Operators, pages 135-170. Birkhäuser Basel, Basel, (2003)

21. Hansen, A.C.: On the solvability complexity index, the $n$-pseudospectrum and approximations of spectra of operators. J. Am. Math. Soc. 24(1), 81-124 (2011)

22. Helffer, B., Mohamed, A.: Asymptotic of the density of states for the Schrödinger operator with periodic electric potential. Duke Math. J. 92(1), 1-60 (1998)

23. Hoang, V., Plum, M., Wieners, C.: A computer-assisted proof for photonic band gaps. Z. Angew. Math. Phys. 60(6), 1035-1052 (2009) 
24. Last, Y.: Spectral theory of sturm-liouville operators on infinite intervals: a review of recent developments. Sturm-Liouville Theory, pp. 99-120 (2005)

25. Mathieu, É.: Mémoire sur le mouvement vibratoire d'une membrane de forme elliptique. J. Math. Pures Appl. 13, 137-203 (1868)

26. Morse, P.M., Feshbach, H.: Methods of theoretical physics. Am. J. Phys. 22(6), 410-413 (1954)

27. Parnovski, L.: Bethe-Sommerfeld conjecture. In: Ann. Henri Poincaré, vol. 9, no. 3, pp. 457-508 (2008)

28. Popov, V.N., Skriganov, M.M.: Remark on the spectrum structure of the two-dimensional Schrödinger operator with the periodic potential. Zap. Nauchnykh Sem. POMI 109, 131-133 (1981)

29. Reed, M., Simon, B.: Methods of modern mathematical physics. IV. Analysis of operators. Academic Press [Harcourt Brace Jovanovich, Publishers], New York, London (1978)

30. Reed, M.,Simon, B.: Methods of modern mathematical physics. I. Functional Analysis. Academic Press, Inc. [Harcourt Brace Jovanovich, Publishers], New York, 2nd edn. (1980)

31. Rofe-Beketov, F.S.: On the spectrum of non-selfadjoint differential operators with periodic coefficients. Dokl. Akad. Nauk SSSR 152, 1312-1315 (1963)

32. Rösler, F.: On the solvability complexity index for unbounded selfadjoint and schrödinger operators. Integral Equ. Oper. Theory 91(6), 54 (2019)

33. Schwinger, J.: On the bound states of a given potential. Proc. Nat. Acad. Sci. U.S.A. 47, 122-129 (1961)

34. Shin, K.C.: On the shape of spectra for non-self-adjoint periodic Schrödinger operators. J. Phys. A 37(34), 8287-8291 (2004)

35. Simon, B.: Notes on infinite determinants of Hilbert space operators. Adv. Math. 24(3), 244-273 (1977)

36. Skriganov, M.M.: The spectrum band structure of the three-dimensional Schrödinger operator with periodic potential. Invent. Math. 80(1), 107-121 (1985)

37. Sommerfeld, A., Bethe, H.: Elektronentheorie der Metalle. In: Aufbau Der Zusammenhängenden Materie. Volume 19 of Heidelberger Taschenbücher, pp. 333-622. Springer, Berlin Heidelberg, Berlin, Heidelberg (1933)

38. Teschl, G.: Jacobi operators and completely integrable nonlinear lattices. Mathematical surveys and monographs . American Mathematical Society (2000)

39. Trefethen, L.N., Embree, M.: Spectra and pseudospectra. The behavior of nonnormal matrices and operators. Princeton University Press, Princeton, NJ (2005)

Publisher's Note Springer Nature remains neutral with regard to jurisdictional claims in published maps and institutional affiliations. 